\title{
Autonomous coupled oscillators with hyperbolic strange attractors
}

\author{
Sergey P. Kuznetsov ${ }^{\mathrm{a}}$, Arkady Pikovsky ${ }^{\mathrm{b}, *}$ \\ ${ }^{a}$ Kotel'nikov Institute of Radio Engineering and Electronics of RAS, Saratov Branch, Zelenaya 38, Saratov, 410019, Russian Federation \\ ${ }^{\mathrm{b}}$ Department of Physics, Potsdam University, 14469 Potsdam, Germany
}

Received 12 January 2007; received in revised form 24 May 2007; accepted 25 May 2007

Available online 8 June 2007

Communicated by J. Stark

\begin{abstract}
We propose several examples of smooth low-order autonomous dynamical systems which have apparently uniformly hyperbolic attractors. The general idea is based on the use of coupled self-sustained oscillators where, due to certain amplitude nonlinearities, successive epochs of damped and excited oscillations alternate. Because of additional, phase sensitive coupling terms in the equations, the transfer of excitation from one oscillator to another is accompanied by a phase transformation corresponding to some chaotic map (in particular, an expanding circle map or Anosov map of a torus). The first example we construct is a minimal model possessing an attractor of the Smale-Williams type. It is a four-dimensional system composed of two oscillators. The underlying amplitude equations are similar to those of the predator-pray model. The other three examples are systems of three coupled oscillators with a heteroclinic cycle. This scheme presents more variability for the phase manipulations: in the six-dimensional system not only the Smale-Williams attractor, but also an attractor with Arnold cat map dynamics near a two-dimensional toral surface, and a hyperchaotic attractor with two positive Lyapunov exponents, are realized.
\end{abstract}

(C) 2007 Elsevier B.V. All rights reserved.

Keywords: Coupled oscillators; Hyperbolic systems; Smale-Williams strange attractor; Arnold cat map

\section{Introduction}

Hyperbolicity is one of the central concepts in the mathematical theory of dynamical chaos. In a hyperbolic attractor all trajectories are of saddle type, all have stable and unstable manifolds of the same dimension, and there are no tangencies between the stable and unstable manifolds. Motions on the strange hyperbolic attractors demonstrate strong chaotic properties and allow a rather complete mathematical description; see, e.g., Ref. [1]. Hyperbolic strange attractors are structurally stable (robust); they can be characterized both topologically (in terms of symbolic dynamics) and probabilistically (in terms of the Sinai-Bowen-Ruelle measures).

There are two popular mathematical examples of lowdimensional hyperbolic strange attractors: the Plykin attractor and the Smale-Williams solenoid [2,1]. The Plykin attractor

\footnotetext{
* Corresponding author. Tel.: +49 3319771472.

E-mail address: pikovsky@ stat.physik.uni-potsdam.de (A. Pikovsky).
}

occurs in a special delicately organized map in a domain on a plane with three holes. The Smale-Williams attractor appears in a mapping of a toroidal domain into itself in phase space of dimension 3 or more.

Until very recently, only geometrical constructions of the hyperbolic strange attractors were known. An important problem to address is: can such constructions be realized by Poincaré maps of systems governed by differential equations allowing a physical realization?

The possibility of hyperbolic strange attractors appearing in systems arising from the perturbation of quasiperiodic motions on tori of dimension 3 and more was discussed by Newhouse et al. [3], although without a concrete example being presented. Attempts to compose a three-dimensional set of ordinary differential equations with an attractor of Plykin type in the Poincaré map have not yet resulted in a construction that could be implemented as a physical system [4,5]. As regards the Smale-Williams solenoids, it was shown that an attractor of such a type may appear as a result of a codimension one bifurcation [6], but again, no explicit examples of differential 

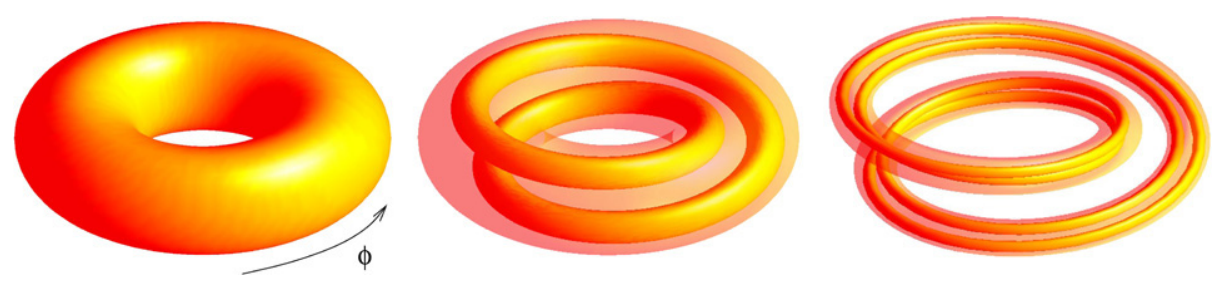

Fig. 1. Construction of the Smale-Williams attractor.
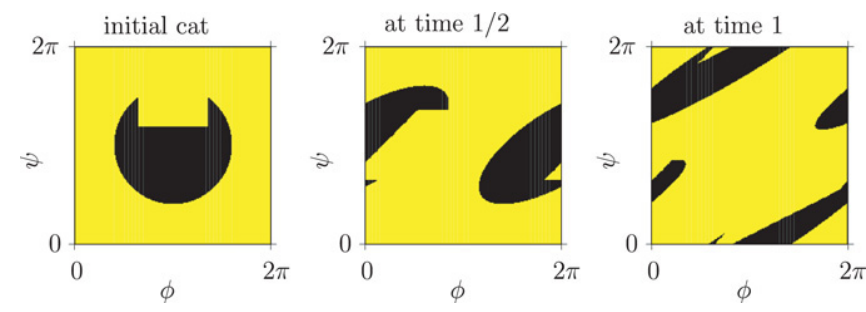

Fig. 2. Illustration of the action of the Arnold cat map.

equations manifesting this phenomenon were delivered.

Quite recently one of us has advanced a physically realistic example of the Smale-Williams attractor in the fourdimensional Poincaré map of a non-autonomous periodically driven system of two coupled self-oscillators [8]. Later, such a system was designed as an electronic device and studied experimentally [9]. Similar constructions of coupled nonautonomous self-oscillators were used to implement dynamics associated with the Arnold cat map [10] and with the robust strange nonchaotic attractor [11].

In the present paper we exploit the approach of Ref. [8] to introduce examples of autonomous coupled oscillator systems with hyperbolic strange attractors. Four models we propose are termed below as A, B, C, and D.

Model A consists of two asymmetrically coupled oscillators. The underlying amplitude equations are similar to those in the predator-pray model. The amplitudes associated with two subsystems become large or small alternately, and the process of the excitation transfer from one subsystem to another and back is accompanied with a doubling of the phase variable, i.e. it corresponds to the expanding circle map (sometimes called the Bernoulli map [12]). In the three-dimensional Poincaré map the system has an attractor of the Smale-Williams type. We stress that this is a flow model of minimal dimension (four) to have such an attractor.

Models B, C, and D are composed each of three coupled autonomous self-oscillators, and the phase space dimension is six. We select the underlying amplitude equations to be symmetric. Choosing a particular structure of the coupling terms responsible for the transfer of the excitation from one oscillator to another, it is possible to arrange not only the Smale-Williams attractor (model B), but also a system possessing an attractor located near a two-dimensional toral surface with dynamics of phases governed by the Arnold cat map (model C), and a system manifesting a hyperchaotic attractor with two positive Lyapunov exponents (model D).

\section{Smale-Williams attractor and toral hyperbolic maps}

The Smale-Williams attractor is considered in most texts on chaos theory (in some of them it is even used as a cover picture). The construction is based on a mapping of a toroidal domain in a three-dimensional space into itself, as illustrated in Fig. 1. One iteration of the map corresponds to a longitudinal stretch of the torus with contraction in the transversal directions, and insertion of the doubly folded "tube" into the original domain. Note that this mapping cannot be produced by a continuous flow in the three-dimensional phase space. A minimal dimension for a continuous-time system to perform such a transformation is four; in such a case the Smale-Williams attractor should occur in the threedimensional Poincaré map.

For the transformation depicted in Fig. 1 an essential property is the doubling of the cyclic coordinate around the torus:

$\phi_{t+1}=2 \phi_{t}$,

which is known as one of the expanding circle maps, or the Bernoulli map [12]. In general, the transformation of the angle variable $\phi$ may be non-uniform, but in any case it must be monotonous and possess the characteristic topological property: one turn of the pre-image by $2 \pi$ is accompanied with a rotation by $4 \pi$ for the image. In other directions, transversal to the coordinate $\phi$, the mapping has to be contracting.

Another textbook example of hyperbolic dynamics is the socalled Arnold cat map (a representative of a general class of Anosov diffeomorphisms of torus)

$$
\left(\begin{array}{l}
\phi_{t+1} \\
\psi_{t+1}
\end{array}\right)=\left(\begin{array}{ll}
2 & 1 \\
1 & 1
\end{array}\right)\left(\begin{array}{l}
\phi_{t} \\
\psi_{t}
\end{array}\right)
$$

defined on a torus $0 \leq \phi<2 \pi, 0 \leq \psi<2 \pi$. Because of the identity

$$
\left(\begin{array}{ll}
2 & 1 \\
1 & 1
\end{array}\right)=\left(\begin{array}{ll}
1 & 1 \\
1 & 0
\end{array}\right)\left(\begin{array}{ll}
1 & 1 \\
1 & 0
\end{array}\right)
$$

this map may be represented as a twofold application of the transformation

$\phi_{t+1 / 2}=\phi_{t}+\psi_{t} \quad(\bmod 2 \pi)$,

$\psi_{t+1 / 2}=\phi_{t} \quad(\bmod 2 \pi)$,

which is equivalent to the Fibonacci map

$\phi_{t+1 / 2}=\phi_{t}+\phi_{t-1 / 2}$.

We illustrate iterations of this map in Fig. 2 using the traditional picture of a cat face.

Eigenvalues of the linear transformation (2) are $(3+$ $\sqrt{5}) / 2 \approx 2.618$ and $(3-\sqrt{5}) / 2 \approx 0.382$, respectively. For 

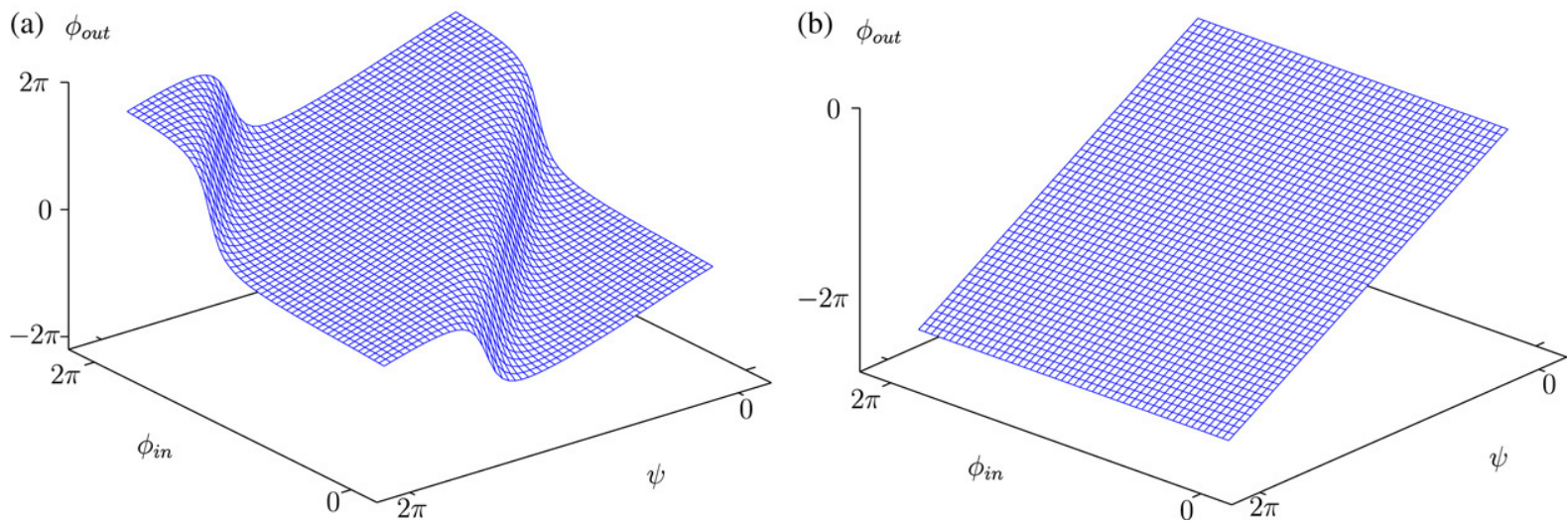

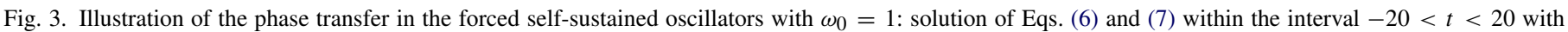
$\epsilon=0.1$ (a) and solution of Eqs. (8) and (9) within the interval $-20<t<20$ with $\Gamma=0.05, \epsilon=0.01$ (b).

every trajectory there is a one-dimensional unstable manifold and a one-dimensional stable manifold; they are associated with the directions of the two eigenvectors. It is worth noting that the map (2) is invertible and area-preserving.

In the class of non-invertible maps on the two-dimensional torus one can introduce a linear map with two eigenvalues larger than 1 in absolute value, e.g.

$$
\left(\begin{array}{l}
\phi_{t+1} \\
\psi_{t+1}
\end{array}\right)=\left(\begin{array}{ll}
1 & 3 \\
1 & 0
\end{array}\right)\left(\begin{array}{l}
\phi_{t} \\
\psi_{t}
\end{array}\right) .
$$

Here the eigenvalues are $(\sqrt{13}+1) / 2 \approx 2.303$ and $(-\sqrt{13}+$ $1) / 2 \approx-1.303$. Each trajectory has two unstable directions, and this situation is called hyperchaos. Similar to the Arnold cat map, this hyperchaotic map can be represented as a composition of simpler transformations with a use of the identity:

$$
\left(\begin{array}{ll}
1 & 3 \\
1 & 0
\end{array}\right)=\left(\begin{array}{ll}
1 & 1 \\
1 & 0
\end{array}\right)\left(\begin{array}{ll}
0 & 1 \\
1 & 0
\end{array}\right)\left(\begin{array}{ll}
0 & 3 \\
1 & 0
\end{array}\right) .
$$

Thus, we can write the hyperchaotic map as a composition of three maps:

$$
\begin{aligned}
& \phi_{t+1 / 3}=3 \phi_{t-1 / 3}, \quad \phi_{t+2 / 3}=\phi_{t}, \\
& \phi_{t+1}=\phi_{t+2 / 3}+\phi_{t+1 / 3} .
\end{aligned}
$$

A common feature of the above mappings is that the discrete-time dynamics is considered in terms of variables that are $2 \pi$-periodic, i.e. they are defined on a circle or on a torus. In the following sections we show how one can implement such mappings with the help of coupled oscillatory systems.

\section{Manipulation of phases of driven oscillations}

In the context of realistic physical systems, we may interpret a cyclic coordinate defined modulo $2 \pi$ as a phase of oscillations. For example, in a self-oscillatory system a particular state on the periodic limit cycle can be characterized by the phase, which is nothing other than the coordinate along the cycle (normalized by $2 \pi$ ). We now describe how one can accomplish a transfer of the phase from one oscillator to another accompanied with its appropriate transformation.
Suppose we have a signal $f(t)$ which is nearly sinusoidal $f(t)=\cos \left(\omega_{0} t+\psi\right)$. At first glance, by forcing a self-sustained oscillator with the signal $f(t)$, one could easily transfer the phase $\psi$ to this oscillator. However, a usual self-sustained oscillator, being forced periodically, although adjusting its phase to that of the external force, does not forget completely its own phase prior to forcing. This is illustrated in Fig. 3(a). The data are obtained from a computer solution of the equations

$\dot{u}=\omega_{0} w+\left(1-u^{2}-w^{2}\right) u+\epsilon f(t)$,

$\dot{w}=-\omega_{0} u+\left(1-u^{2}-w^{2}\right) w$,

describing a self-sustained oscillator with frequency $\omega_{0}$ and unit amplitude of the limit cycle, driven by the signal $f(t)$. Starting with the limit cycle at $t=-20$ with the initial phase of the oscillations $\phi_{\text {in }}=\arctan \frac{w(t=-20)}{u(t=-20)}$, we integrate Eqs. (6) and (7), and at $t=20$ calculate the resulting phase $\phi_{\text {out }}=\arctan \frac{w(t=20)}{u(t=20)}$. Observe a nontrivial dependence of the output phase $\phi_{\text {out }}$ both on the phase of the forcing $\psi$ and on the initial phase $\phi_{\text {in }}$. Here we do not have the phase transfer of the required topological class.

A mechanism that gives us the possibility to obtain the phase doubling consists of the following [8]. Consider a self-oscillator undergoing a transition from damped to excited state due to a slow variation of the parameter responsible for the birth of the limit circle (the supercritical Andronov-Hopf bifurcation). While the amplitude of the oscillator in the damped stage is extremely small, the forcing $f(t)$ dominates, and the phase of oscillations is $\psi+$ const. As the parameter varies, the oscillator becomes active, the amplitude of the limit cycle grows, and the phase preserves the shift $\psi$.

Let us demonstrate the phase transfer from the driving force to the oscillator. For this, we arrange the parameter responsible for the growth rate of small oscillations in Eqs. (6) and (7), to vary from negative to positive values:

$\dot{u}=\omega_{0} w+\left(\Gamma t-u^{2}-w^{2}\right) u+\epsilon f(t)$,

$\dot{w}=-\omega_{0} u+\left(\Gamma t-u^{2}-w^{2}\right) w$,

where the coefficient $0<\Gamma \ll 1$. For $t \rightarrow-\infty$ the oscillator is highly damped, and the forced solution is $u \approx-\epsilon f(t) / \Gamma t$, 

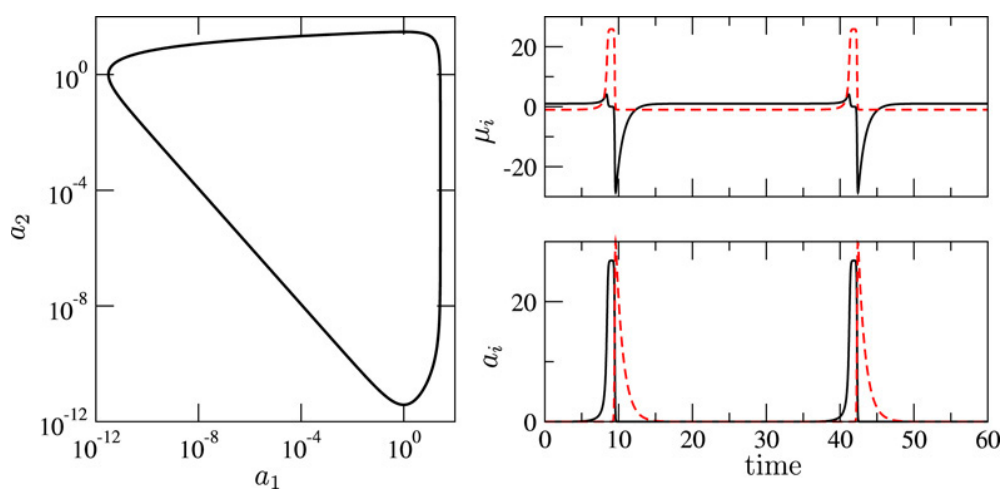

Fig. 4. The phase portrait of the system (10) (left panel), the time courses of the variables $a_{i}$ and the growth rates $\mu_{i}$ (right panel). Black solid line: $i=1$; red short dashed line: $i=2$. (For interpretation of the references to colour in this figure legend, the reader is referred to the web version of this article.)

$w \approx 0$. For large positive times the amplitude of oscillations is large $u^{2}+w^{2} \approx \Gamma t$, but the phase remains that of $f(t)$, i.e. $\psi+$ const. We illustrate the mechanism of the phase transfer in Fig. 3(b). Again, the process started at $t=-20$ and ended at $t=20$. Now one can see that the output phase $\phi_{\text {out }}$ does not depend on the initial phase $\phi_{\text {in }}$ but depends linearly on the phase of the forcing $\psi$, i.e. $\phi_{\text {out }}=\psi+$ const.

In our example the phase transfer takes place in a situation of resonant excitation of the oscillator in the course of its passage from the damped to the active stage. Numerics show that the resonance condition is not necessary to achieve the desired phase transfer. Even with a large difference between the driving frequency and that of the oscillator, the pictures similar to Fig. 3(b) can be observed in a wide range of the forcing amplitudes $\epsilon$ and the rates of the parameter variation $\Gamma$.

On the basis of the phase transformation $\psi \rightarrow \phi$ in the model (8) and (9) we can easily construct any linear transformation $A \psi_{1} \pm B \psi_{2} \rightarrow \phi$, with positive integers $A$ and $B$. Indeed, if we have processes $x_{1}=\cos \left(\omega_{0} t+\psi_{1}\right)$ and $x_{2}=\cos \left(\omega_{0} t+\psi_{2}\right)$, we may use the signal $f(t)=$ $x_{1}^{A} x_{2}^{B}$, which has spectral components at frequencies $(A \pm$ $B) \omega_{0}$ with the phases $A \psi_{1} \pm B \psi_{2}$. In the case of the nonresonant excitation transfer, we have to ensure prevalence of the spectral component possessing the desired phase. It may be accomplished, e.g., by means of careful selection of particular expressions for the driving terms in the equations combining $x_{1}, \dot{x}_{1}, x_{2}, \dot{x}_{2}$. For example, given process $x=\cos \left(\omega_{0} t+\psi\right)$, we obtain a pure signal with the doubled phase by choosing $f(t)=x \dot{x}=-\frac{1}{2} \omega_{0} \sin \left(2 \omega_{0} t+2 \psi\right)$.

For simplicity of presentation, we have considered above the phase transfer from purely sinusoidal signals. However, it is clear that the mechanism holds also for any signal allowing the sine approximation on some finite time interval, which contains the moment of the rate change sign in the self-oscillator driven by this signal; outside of this interval the time dependence for the signal can be arbitrary. In paricular, in Fig. 3 the integration is in the range $-20<t<20$; this means that the form of the signal outside this interval is irrelevant. Thus, the mechanism will work for signals consisting of nearly sinusoidal patches, like those in Fig. 11.

\section{Coupled oscillators with alternating excitation}

In this section we construct autonomous coupled oscillator models that naturally demonstrate alternations between decay and growth of the amplitudes of the oscillations. With some additional terms in the equations we will be able to ensure exchange of the excitation between the oscillators accompanied by the phase evolution governed by chaotic maps discussed in Section 2.

Let us start with the modified predator-pray equations written down in terms of strictly non-negative variables $a_{1}$ and $a_{2}$ (which may by interpreted as the pray and predator populations):

$\dot{a}_{1}=\mu_{1} a_{1}, \quad \dot{a}_{2}=\mu_{2} a_{2}$,

where

$\mu_{1}=1-a_{2}+\frac{1}{2} a_{1}-\frac{1}{50} a_{1}^{2}, \quad \mu_{2}=a_{1}-1$.

The system has a limit cycle which passes very close to the saddle fixed point at the origin $a_{1}=0, a_{2}=0$, and goes around the unstable focus at $a_{1}=1, a_{2}=\frac{37}{25}$ (Fig. 4). During this process, the growth rates $\mu_{1,2}$ alternately change sign.

Now, let us regard $a_{1}$ and $a_{2}$ as squared amplitudes of two oscillators and represent them as $a_{i}=x_{i}^{2}+y_{i}^{2}$, where $x_{i}$, $y_{i}$ are some new variables undergoing oscillations with some frequency $\omega_{0}$. Specifically, we set

$\dot{x}_{1}=\omega_{0} y_{1}+\mu_{1} x_{1}, \quad \dot{x}_{2}=\omega_{0} y_{2}+\mu_{2} x_{2}$,

$\dot{y}_{1}=-\omega_{0} x_{1}+\mu_{1} y_{1}, \quad \dot{y}_{2}=-\omega_{0} x_{2}+\mu_{2} y_{2}$,

where $\mu_{1,2}$ are defined by the relations (11). As the values $a_{i}=x_{i}^{2}+y_{i}^{2}$ obey the Eq. (10), we have a situation where two oscillators alternately undergo growth and decay. In (12) the phases of two oscillators are uncoupled. Below, in the next section, we will introduce additional coupling to ensure the phase transfer from one to another oscillator in the course of this process.

Now let us turn to a system of three coupled elements governed by the equations

$\dot{a}_{1}=\mu_{1} a_{1}, \quad \dot{a}_{2}=\mu_{2} a_{2}, \quad \dot{a}_{3}=\mu_{3} a_{3}$, 
where the variables $a_{i}$ are assumed to be non-negative, and

$$
\begin{aligned}
& \mu_{1}=1-a_{1}-\frac{1}{2} a_{2}-2 a_{3}, \\
& \mu_{2}=1-a_{2}-\frac{1}{2} a_{3}-2 a_{1}, \\
& \mu_{3}=1-a_{3}-\frac{1}{2} a_{1}-2 a_{2} .
\end{aligned}
$$

Note a symmetry with respect to a cyclic transposition of the variables $a_{i}$. There are three saddle equilibrium points $\left(a_{1}=\right.$ $\left.1, a_{2,3}=0\right),\left(a_{2}=1, a_{1,3}=0\right)$, and $\left(a_{3}=1, a_{1,2}=0\right)$ connected by orbits forming the so-called heteroclinic cycle or contour (Fig. 5). An arbitrary initial state of this system evolves in such a way that the orbit approaches the contour ever more closely. Visiting the neighborhoods of the saddle points, it resides there for longer and longer (infinitely growing) time intervals. (See, e.g., [13] for a detailed description of the heteroclinic cycle.)

Again, we introduce the oscillatory variables $x_{i}, y_{i}, i=$ $1,2,3$, and set $a_{i}=x_{i}^{2}+y_{i}^{2}$. Then, we write down the evolution equations for the oscillating variables $x_{i}$ and $y_{i}$ with frequencies $\omega_{i}$ as follows:

$$
\begin{aligned}
& \dot{x}_{1}=\omega_{1} y_{1}+\mu_{1} x_{1}, \quad \dot{x}_{2}=\omega_{2} y_{2}+\mu_{2} x_{2}, \\
& \dot{x}_{3}=\omega_{3} y_{3}+\mu_{3} x_{3}, \\
& \dot{y}_{1}=-\omega_{1} x_{1}+\mu_{1} y_{1},
\end{aligned}
$$

Oscillations in these three subsystems alternately grow and decay in accordance with Fig. 5. The quantities $\mu_{i}$ determine the growth rates of the amplitudes $a_{i}$ and vary during the evolution in the range $-1<\mu<0.5$. In the next section we will consider several ways of adding coupling terms in Eq. (15) to obtain different variants of the phase transfer between the alternately exciting oscillators.

\section{Coupled oscillators with hyperbolic attractors}

This is the main section of the paper. We will formulate oscillator models possessing the hyperbolic attractors. Each model consists of two or three oscillators, which become excited alternately because of the appropriate mutual amplitude nonlinearity. At the beginning of an active stage, each oscillator adopts the phase of excitation from its partners, with a transformation corresponding to a certain chaotic map. This excitation transfer is ensured by additional terms introduced in the equations at the final step of our construction of the models. As basic tools for computer study of the dynamics we will exploit Poincaré maps and iteration diagrams illustrating the phase transformation in the course of the excitation transfer, calculation of the Lyapunov exponents, and characterization of the processes with correlation functions.

\subsection{Model A: Smale-Williams attractor in a system of two coupled oscillators}

Let us complement the model (12) with additional coupling terms in the first equations for the oscillators 1 and 2 proportional to $x_{2} y_{2}$ and $x_{1}$, respectively, and arrive at the following model $A$ :

$$
\begin{aligned}
& \dot{x}_{1}=\omega_{0} y_{1}+\left(1-a_{2}+\frac{1}{2} a_{1}-\frac{1}{50} a_{1}^{2}\right) x_{1}+\varepsilon x_{2} y_{2}, \\
& y_{1}=-\omega_{0} x_{1}+\left(1-a_{2}+\frac{1}{2} a_{1}-\frac{1}{50} a_{1}^{2}\right) y_{1}, \\
& \dot{x}_{2}=\omega_{0} y_{2}+\left(a_{1}-1\right) x_{2}+\varepsilon x_{1}, \\
& \dot{y}_{2}=-\omega_{0} x_{2}+\left(a_{1}-1\right) y_{2},
\end{aligned}
$$

where

$a_{1}=x_{1}^{2}+y_{1}^{2}, \quad a_{2}=x_{2}^{2}+y_{2}^{2}$.

and $\varepsilon$ is the coupling constant. In the numerical investigation below, we fix the parameters $\omega_{0}=2 \pi, \varepsilon=0.3$. For this parameter set, we checked different initial conditions and observed that all of them lead to the same attractor.

Now, in the course of transition of the second oscillator to the active stage, it is forced by the term $x_{1}$ and adopts the phase from the first oscillator. In turn, the first oscillator at the transition to the active stage is forced by the term $x_{2} y_{2}$. Hence, in accordance with the mechanism explained in Section 3, the phase doubling will occur during the transfer of the excitation.

Fig. 6 shows typical time dependences for the variables $x_{1,2}$, $y_{1,2}$ obtained from computer solution of Eq. (16). Amplitudes of two oscillators alternate, approximately like those in Fig. 4.

Let us demonstrate that the phase doubling indeed takes place. To do this, one can construct the Poincaré map in the following way. In the four-dimensional phase space $\left\{x_{1}, y_{1}, x_{2}, y_{2}\right\}$ we define a surface $S=x_{1}^{2}+y_{1}^{2}-x_{2}^{2}-y_{2}^{2}=0$ (it corresponds to a condition of equality of the instantaneous amplitudes for two oscillators). Then, we consider intersections of this surface with trajectories in the direction of the growing value of $S$. Let an initial point on the surface $S$ be associated with a three-dimensional vector $\mathbf{v}_{n}$, and the next intersection of the surface in the same direction yield the image vector $\mathbf{v}_{n+1}$. Thus, we have a three-dimensional Poincaré map $\mathbf{v}_{n+1}=$ $\mathbf{T}\left(\mathbf{v}_{n}\right)$.

In accordance with the computations, at the selected parameter values the average time interval between the intersections of the surface $S=0$ is approximately $T_{\mathrm{av}}=$ 7.245. It is a characteristic time interval between the bursts in Fig. 6.

At successive intersections of the surface $S=0$ we define the phases of the second oscillator as $\varphi=\arg \left(x_{2}+\mathrm{i} y_{2}\right)$ and plot them on the diagram $\varphi_{n+1}$ versus $\varphi_{n}$. It is shown in Fig. 7. Clearly, it looks topologically similar to the expanding circle $\operatorname{map} \varphi_{n+1} \approx 2 \varphi_{n}+$ const.

Fig. 8 shows two projections of the attractor for the Poincare map. In fact, both portraits possess a Cantor-like transversal structure, but only one level of it is visually distinguishable on the panel (a).

We may imagine a toroidal domain in the three-dimensional phase space of the Poincare map, where the phase $\varphi$ corresponds to the angle counted around the "hole" of the torus. Application of the map $\mathbf{T}$ corresponds to a twofold longitudinal stretch of the torus, transversal compressing, and embedding as 
(a) $a_{3}$
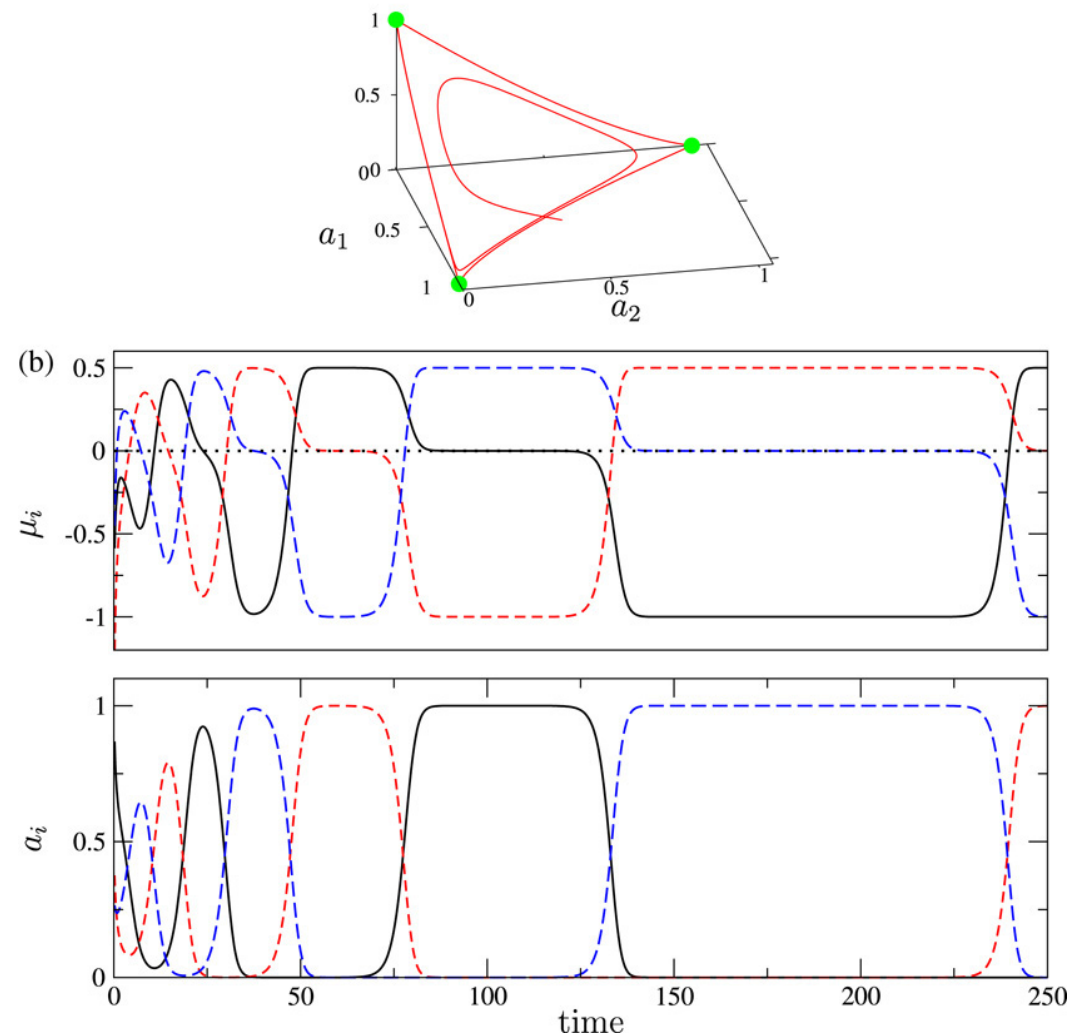

Fig. 5. (a) A heteroclinic contour in the system (13). (b) State variables $a_{i}$ versus time; observe oscillations with a growing period. The corresponding local growth rates alternate between positive and negative values. Black solid line: $i=1$; red short dashed line: $i=2$; blue long dashed line: $i=3$. (For interpretation of the references to colour in this figure legend, the reader is referred to the web version of this article.)
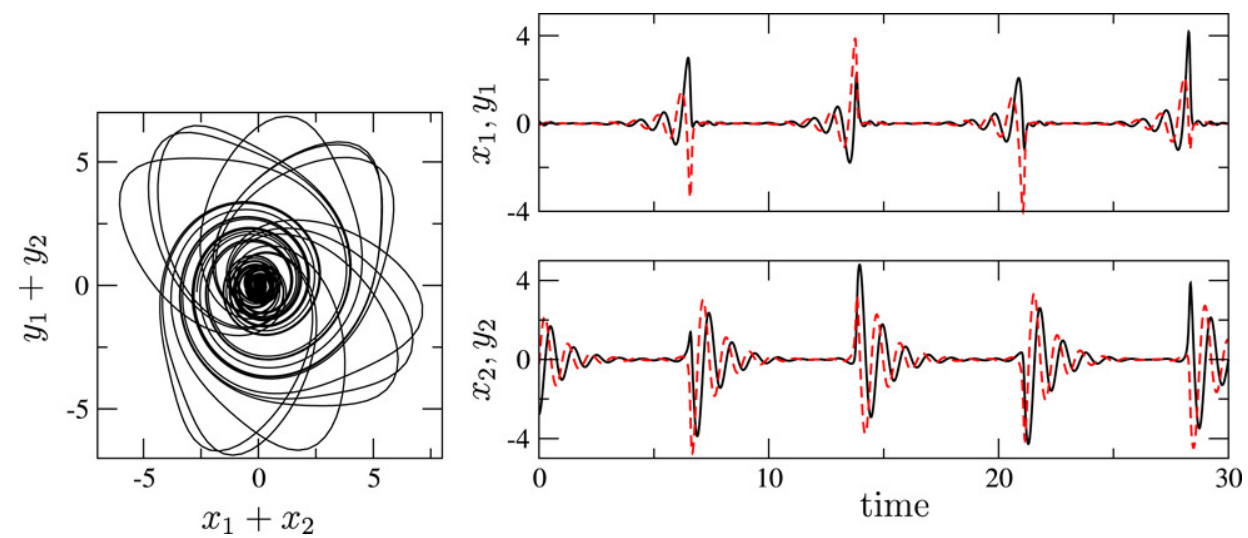

Fig. 6. Oscillations described by Eq. (16) with parameter values $\omega_{0}=2 \pi, \varepsilon=0.3$. Variables $y_{j}$ are shown with dashed red lines. Left panel: a projection on the plane $\left(x_{1}+x_{2}, y_{1}+y_{2}\right)$. (For interpretation of the references to colour in this figure legend, the reader is referred to the web version of this article.)

the doubly folded "tube" inside the original domain. Thus, the attractor of the map $\mathbf{T}$ is just a hyperbolic chaotic attractor of the Smale-Williams type.

Lyapunov exponents for the model $\mathrm{A}$ at the chosen parameter values have been computed using a standard method of Benettin et al. (see, e.g., [14]) by means of a simultaneous solution of Eq. (16) and the respective linearized equations for variations. The results are

$\lambda_{1}=0.0918 \pm 0.0002$, $\lambda_{2}=-0.000004 \pm 0.00003$

$\lambda_{3}=-4.074 \pm 0.002$,

$\lambda_{4}=-4.3936 \pm 0.0005$.

The positive exponent $\lambda_{1}$ indicates the presence of chaos, while the zero exponent $\lambda_{2}$ corresponds to a perturbation associated with a shift along the orbit. The other two Lyapunov exponents are negative and are responsible for the approach of trajectories to the attractor. 


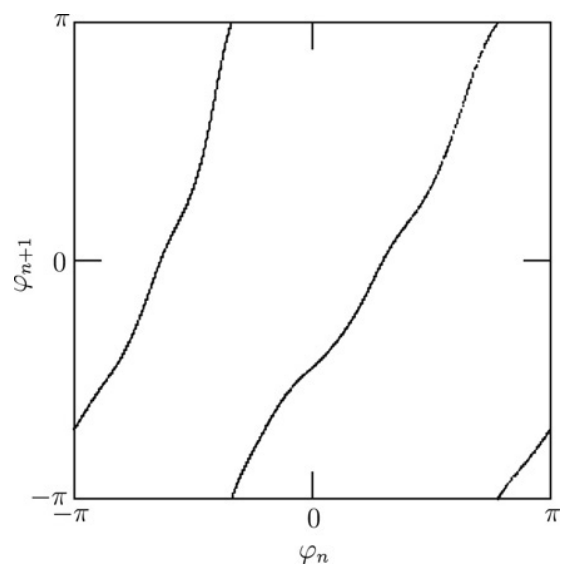

Fig. 7. Iteration diagram for the phases of the second oscillator at successive intersection of the surface $S$ in model (16) with the same parameters as in Fig. 6.

For the Poincaré map, the Lyapunov exponents may be obtained with a relation $\Lambda=\lambda T_{\mathrm{av}}$ (with exclusion of a zero one). The largest Lyapunov exponent is close to the value expected from the approximation based on the expanding circle map (1). Indeed, $\rho_{1}=\exp \left[\lambda_{1} T_{\mathrm{av}}\right]=1.945$ that is close to the factor 2 , which would correspond to the uniform phase doubling.

From the calculated Lyapunov exponents, we can estimate the Lyapunov dimension of the attractor with the Kaplan-Yorke formula [14]. It yields

$D_{L}=m+\frac{\sum_{i=1}^{m} \lambda_{i}}{\left|\Lambda_{m+1}\right|} \approx 2.0225$,

where $m=2$. Respectively, for the attractor of the Poincaré map we obtain

$d=D-1=1.0225$.

Fig. 9 shows a three-dimensional portrait of the attractor of the flow system in the space $\left(x_{2}, y_{2}, a_{1}\right)$. Rotation of the orbit approximately in the horizontal plane corresponds to an epoch

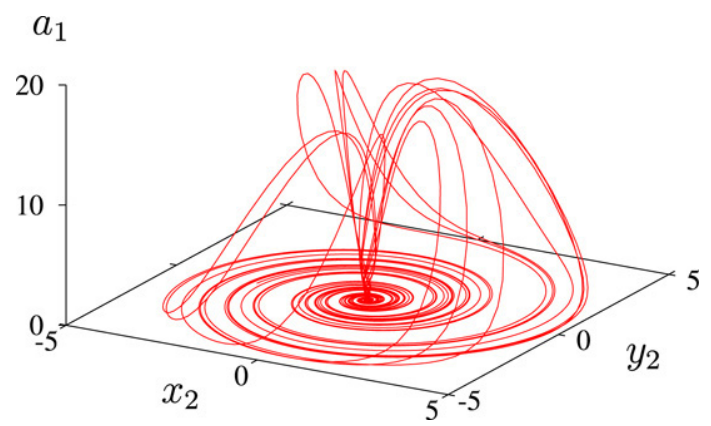

Fig. 9. Portrait of the attractor of the model A (parameters $\omega_{0}=2 \pi, \varepsilon=0.3$ ) in the three-dimensional space $\left(x_{2}, y_{2}, a_{1}=x_{1}^{2}+y_{1}^{2}\right)$. It is worth noting that the fixed point at the origin, at which the orbits pass nearby, possess the stable and unstable manifolds of dimension 2 (in contrast to those well-understood Shilnikov situations [7], where one of the manifolds is one-dimensional). In the picture the first oscillator is characterized only by real amplitude, so information on its phases is lost in this graphical representation.

of excitation and slow decay for the second oscillator and of silence for the first one. The excitation of the first oscillator gives rise to the departure of the orbit from the origin with the subsequent excitation of the second oscillator, its decay, and the next return into a neighborhood of the origin.

In Fig. 10 we show a plot of the autocorrelation function for the observable $x_{1}+x_{2}$. One can see a rather fast decay of correlations. However, the tail of the autocorrelation function has slowly decreasing bursts at times $\approx n T_{\mathrm{av}}$, where $T_{\mathrm{av}}$ is a characteristic period for the amplitudes $a_{1,2}$. The mentioned feature is linked apparently with the fact that the amplitude dynamics at $\varepsilon=0$ is exactly periodic; at finite values of $\varepsilon$, although chaos occurs, the correlations at a former period remain relatively strong, at least for the amplitudes.

\subsection{Model B: Smale-Williams attractor in a perturbed heteroclinic cycle}

Now, let us turn to the model of three oscillators with a heteroclinic cycle (see Fig. 5 end Eq. (15)). To construct the model $B$ we set all the frequencies of the oscillators to be equal:
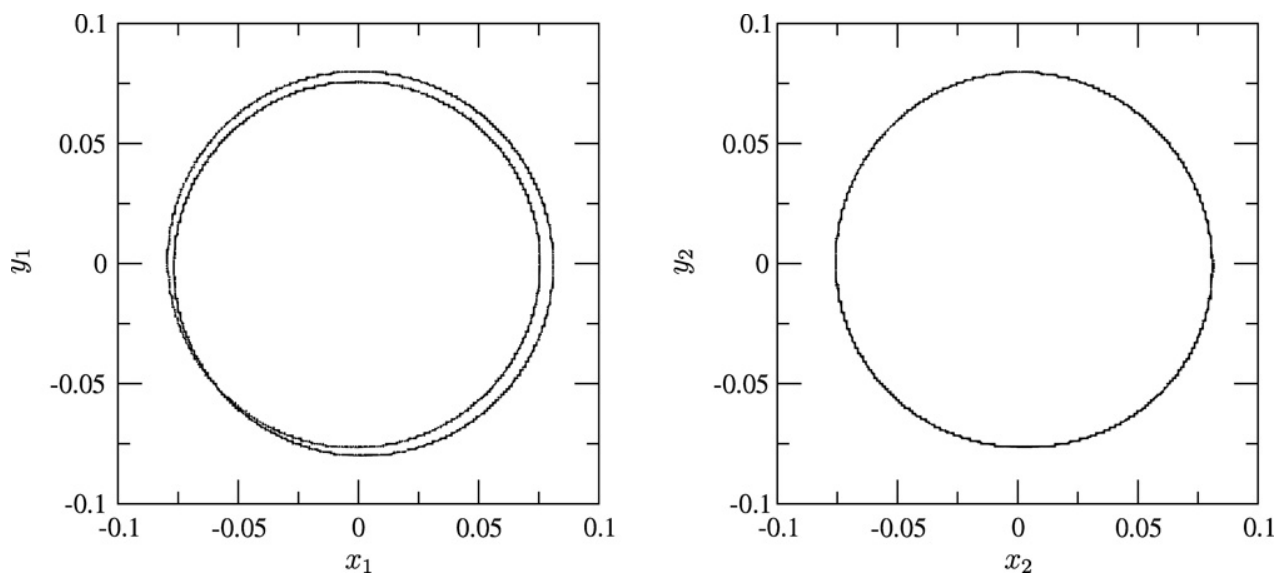

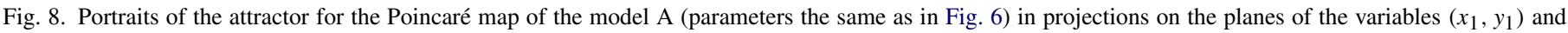
$\left(x_{2}, y_{2}\right)$. 


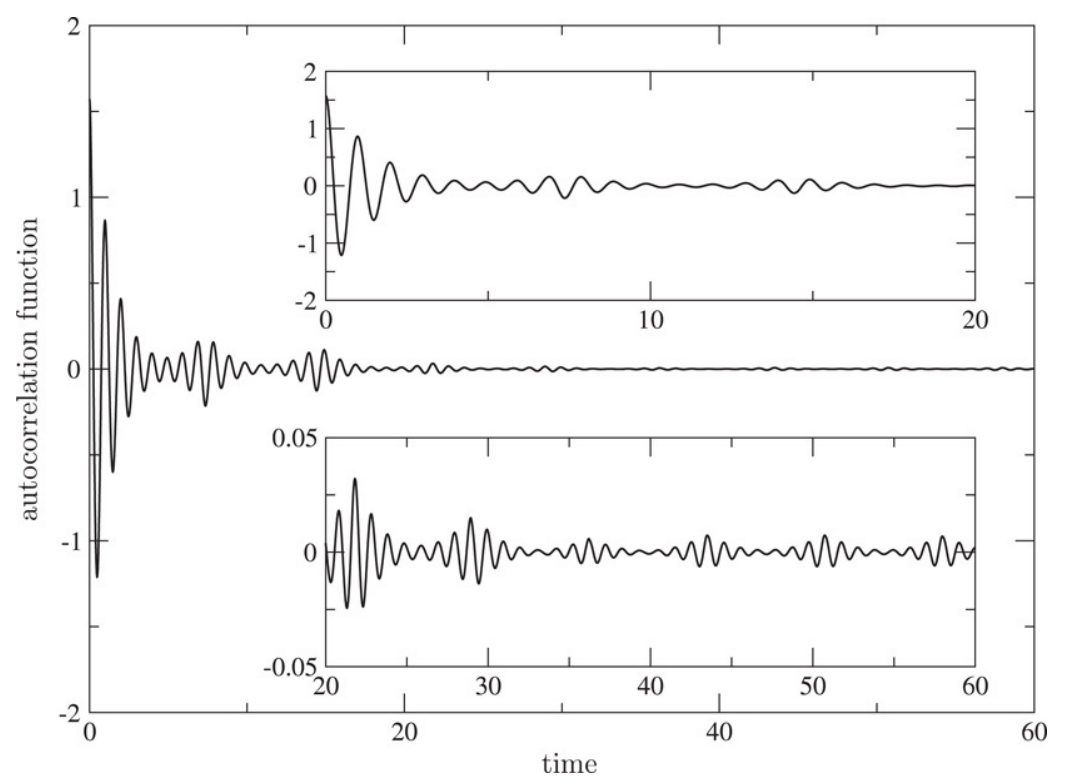

Fig. 10. Autocorrelation function of the observable $x_{1}+x_{2}$ for the model A (parameter values the same as in Fig. 6). Insets show the regions of small time lags and of the correlation tail.

$\omega_{1,2,3}=\omega_{0}$, and add the coupling terms proportional to $x_{2} y_{2}$, $x_{3} y_{3}, x_{1} y_{1}$ in the first equation for each oscillator:

$\dot{x}_{1}=\omega_{0} y_{1}+\left(1-a_{1}-\frac{1}{2} a_{2}-2 a_{3}\right) x_{1}+\varepsilon x_{2} y_{2}$,

$\dot{y}_{1}=-\omega_{0} x_{1}+\left(1-a_{1}-\frac{1}{2} a_{2}-2 a_{3}\right) y_{1}$,

$\dot{x}_{2}=\omega_{0} y_{2}+\left(1-a_{2}-\frac{1}{2} a_{3}-2 a_{1}\right) x_{2}+\varepsilon x_{3} y_{3}$,

$\dot{y}_{2}=-\omega_{0} x_{2}+\left(1-a_{2}-\frac{1}{2} a_{3}-2 a_{1}\right) y_{2}$,

$\dot{x}_{3}=\omega_{0} y_{3}+\left(1-a_{3}-\frac{1}{2} a_{1}-2 a_{2}\right) x_{3}+\varepsilon x_{1} y_{1}$,

$\dot{y}_{3}=-\omega_{0} x_{3}+\left(1-a_{3}-\frac{1}{2} a_{1}-2 a_{2}\right) y_{3}$,

where

$a_{1}=x_{1}^{2}+y_{1}^{2}, \quad a_{2}=x_{2}^{2}+y_{2}^{2}, \quad a_{3}=x_{3}^{2}+y_{3}^{2}$,

and $\varepsilon$ is the coupling constant.

The added terms serve as germs for the excitation of each oscillator at the beginning of the epochs of its activity. It takes place just at each passage of the orbit near the fixed points on the heteroclinic cycle. The oscillators become excited following the order $1 \rightarrow 3 \rightarrow 2 \rightarrow 1 \rightarrow \cdots$. Each new transfer of the excitation is accompanied by the phase doubling in accordance with the mechanism discussed in Section 3. In the computations we fix $\omega_{0}=1$ and $\varepsilon=0.03$. Similarly to the model A, we observe only one attractor testing different initial conditions.

Fig. 11(a) shows typical time dependences for $x_{1}, x_{2}$, and $x_{3}$, obtained from the computer solution of Eq. (17). Observe that in the course of time evolution, three oscillators become

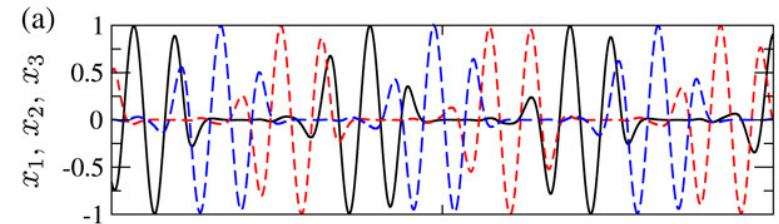

(b)

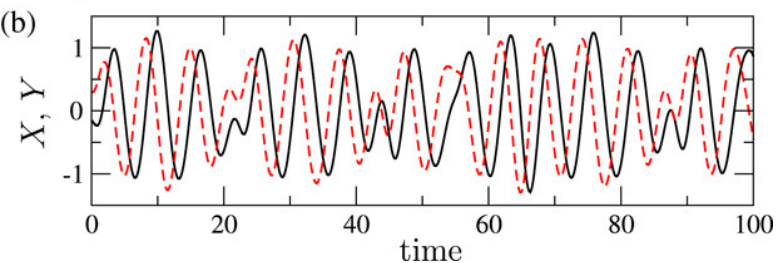

Fig. 11. (a): Time dependences for $x_{1}$ (black solid line), $x_{2}$ (short dashed red line), and $x_{3}$ (long dashed blue line), for model (17) with $\omega_{0}=1$ and $\varepsilon=0.03$. (b): Time dependencies of the symmetrized variables $X=x_{1}+x_{2}+x_{3}$ and $Y=y_{1}+y_{2}+y_{3}$. (For interpretation of the references to colour in this figure legend, the reader is referred to the web version of this article.)

excited one after another. The process is evidently not periodic, although there is a rather well-defined mean period of the amplitude dynamics (in contrast to the model without the added coupling terms, cf. Fig. 5). In panel (b) we show the time dependences for the symmetrized variables $X=x_{1}+x_{2}+x_{3}$ and $Y=y_{1}+y_{2}+y_{3}$. They demonstrate epochs of relatively regular oscillations (2-3 periods), intermingled with transitions that sometimes appear like phase slips. The latter can be seen clearly on the plane $(X, Y)$, where they correspond to pieces of the trajectory passing near the origin (see Fig. 12).

To give evidence for the doubling phase mechanism, let us construct the Poincaré map; it will be done with some additional modification taking into account the symmetry of the system; namely, in the six-dimensional phase space $\left\{x_{1}, y_{1}, x_{2}, y_{2}, x_{3}, y_{3}\right\}$ we define three surfaces determined by 


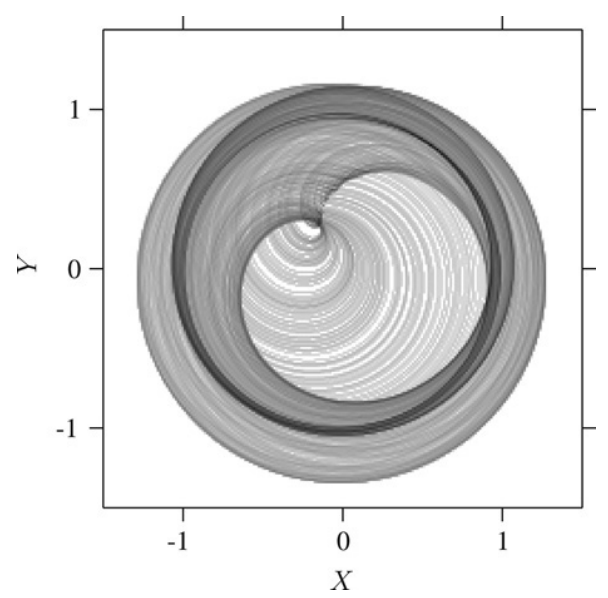

Fig. 12. Projection of the attractor in the model B with $\omega_{0}=1$ and $\varepsilon=0.03$ on the plane $(X, Y)$. We use a gray coding proportional to the density of trajectories to highlight often-visited regions in the phase space.

equations

$S_{1}=x_{1}^{2}+y_{1}^{2}-x_{2}^{2}-y_{2}^{2}=0$,

$S_{2}=x_{2}^{2}+y_{2}^{2}-x_{3}^{2}-y_{3}^{2}=0$,

$S_{3}=x_{3}^{2}+y_{3}^{2}-x_{1}^{2}-y_{1}^{2}=0$,

and examine successive intersections of these surfaces in the direction of growth of $S_{j}$. The intersection of the surface $S_{j}=0$ by a trajectory corresponds to the condition that an amplitude of the rising oscillator $\left(x_{j}, y_{j}\right)$ overtakes that of the previously excited partner. The intersections follow in the order $S_{1} \rightarrow$ $S_{3} \rightarrow S_{2} \rightarrow \cdots$.

Being given a point on a surface $S_{j}=0, j=1,2,3$, it is associated with a five-dimensional vector $\mathbf{v}_{n}$. On the next intersection of a surface $S$ by the trajectory started at $\mathbf{v}_{n}$, we obtain a new vector $\mathbf{v}_{n+1}=\mathbf{T}\left(\mathbf{v}_{n}\right)$. The spaces of images and pre-images may be identified because of the cyclic symmetry of the system. The usual Poincaré map will correspond to a threefold iteration of the map $\mathbf{T}$.

From the numerical simulations, we have estimated the average time interval between the intersection of the surfaces $S_{j}=0$, the value $T_{\mathrm{av}}=10.96$. For a sequence of successive intersections of the surfaces $S_{j}=0$ at time instants $t_{n}$ we define the phases of the oscillations as $\varphi_{n}=\arg \left(x_{j}+\mathrm{i} y_{j}\right)$. The diagram $\varphi_{n+1}$ versus $\varphi_{n}$ is shown in Fig. 13. The presence of the phase doubling mechanism is evident from the picture: the diagram looks topologically similar to the expanding circle map (1).

Fig. 14 shows the projections of the attractor in the Poincaré map onto three planes of the variables $\left(x_{j}, y_{j}\right)$ corresponding to three partial oscillators. Because of strong transversal compression, the fractal structure of the attractor is not distinguishable for the oscillators $j$ (a) and $j+1$ (b) whose amplitudes are equal at the condition of the Poincaré section $S_{j}=0$, but can be seen for the third oscillator $j+2$ (c) whose amplitude is small at this moment in time.

Furthermore, we calculated the Lyapunov exponents for the system (17):

$\lambda_{1}=0.06305 \pm 0.00004$,

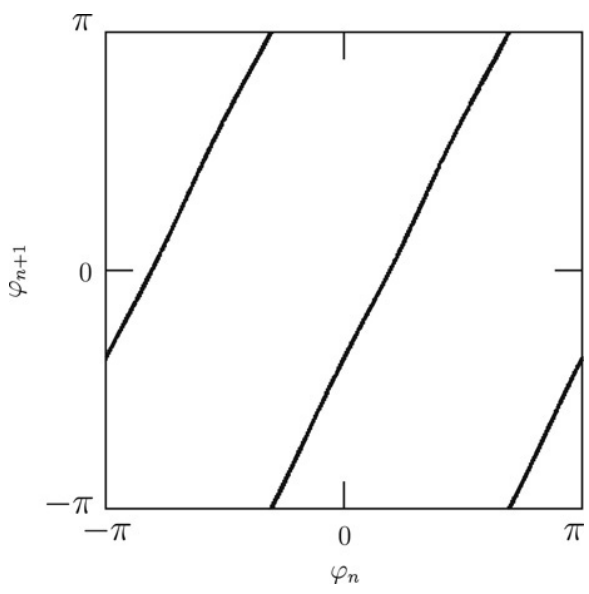

Fig. 13. The mapping $\varphi_{n} \rightarrow \varphi_{n+1}$ obtained numerically for the model (17) with $\omega_{0}=1$ and $\varepsilon=0.03$.

$\lambda_{2}=-0.000001 \pm 0.00001$,

$\lambda_{3}=-0.2101 \pm 0.0002$,

$\lambda_{4}=-0.2259 \pm 0.0004$,

$\lambda_{5}=-0.3395 \pm 0.0006$,

$\lambda_{6}=-1.9999349 \pm 0.0000005$.

One Lyapunov exponent is positive, the next one is close to zero (it corresponds, clearly, to the perturbation directed along the orbit), and the remaining four are negative.

For the map T, the Lyapunov exponents may be expressed via the non-zero exponents of the flow system with the formula $\Lambda=\lambda T_{\mathrm{av}}$. Note that the value of the positive Lyapunov exponent agrees well with that expected from the approximation by the expanding circle map (with factor 2). Indeed, $\exp \left[\lambda_{1} T_{\mathrm{av}}\right]=1.996$, which is very close to 2 .

Calculation of the attractor dimension from the KaplanYorke formula yields for the flow system

$D=m+\frac{\sum_{i=1}^{m} \lambda_{i}}{\left|\lambda_{m+1}\right|}=2+\frac{\lambda_{1}}{\left|\lambda_{3}\right|} \approx 2.30$.

For the attractor of the Poincare map $\mathbf{T}$ the Lyapunov dimension is $d=D-1=1.30$. As follows from the iteration diagram for the phase variables of Fig. 13, application of the map $\mathbf{T}$ implies a twofold stretch in the phase space along the phase variable in the five-dimensional phase space, while in other directions the evolution is accompanied by compression as the respective Lyapunov exponents are negative. This observation confirms that the map $\mathbf{T}$ has a hyperbolic chaotic attractor of the Smale-Williams type.

To characterize statistical properties of the attractor, we calculated the autocorrelation function for the observable $X=x_{1}+x_{2}+x_{3}$ (Fig. 15). The correlations decay rather fast; however, as the inset in Fig. 15 demonstrates, there exists a slowly decaying nearly periodic tail. Its origin is the same as that in Fig. 10: although phases of oscillators are strongly chaotic, the variations of the amplitudes appear to be close to periodic, with a slow decay of correlations. A 
(a)
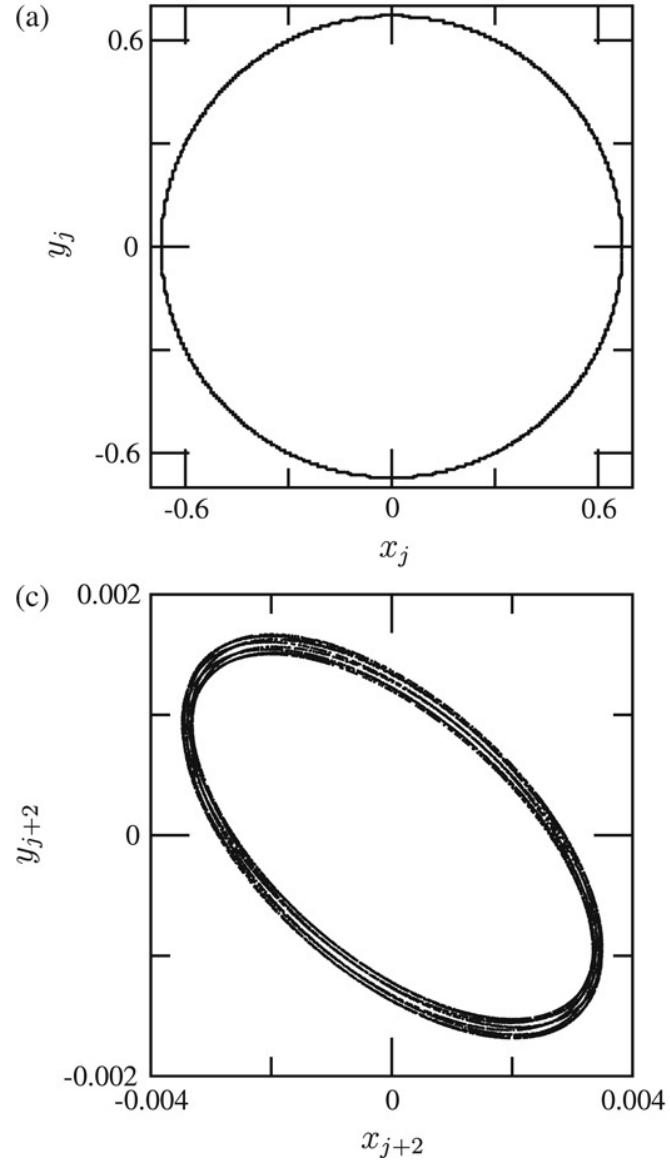

(b)

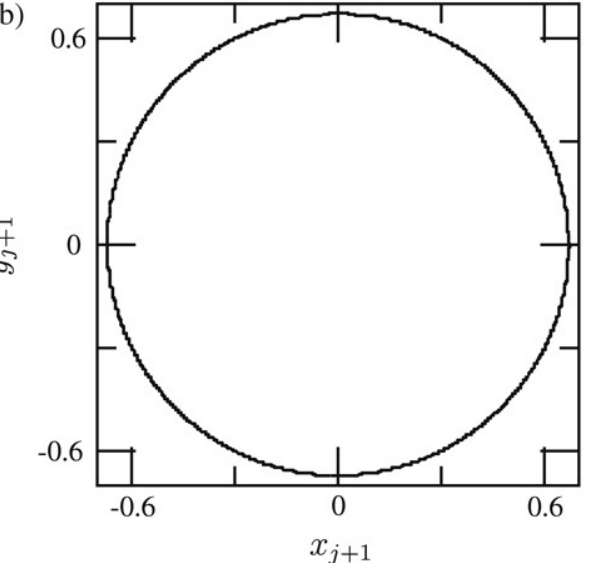

(d)

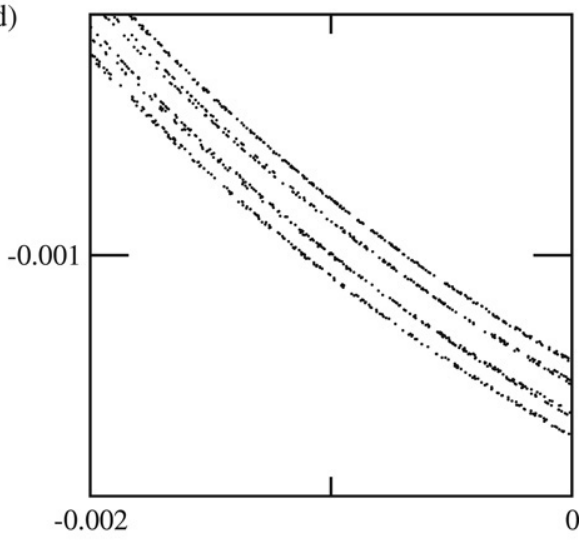

Fig. 14. Portraits of the attractor in the Poincare map of model B (the same parameters as in Fig. 11) in projections onto planes of the variables $\left(x_{j}, y_{j}\right)$, $\left(x_{j+1}, y_{j+1}\right)$, and $\left(x_{j+2}, y_{j+2}\right)$ (note that the indices should be taken as modulo 3). Panel (d) shows an enlargement of a region of panel (c) to make the fractal transverse structure visible.

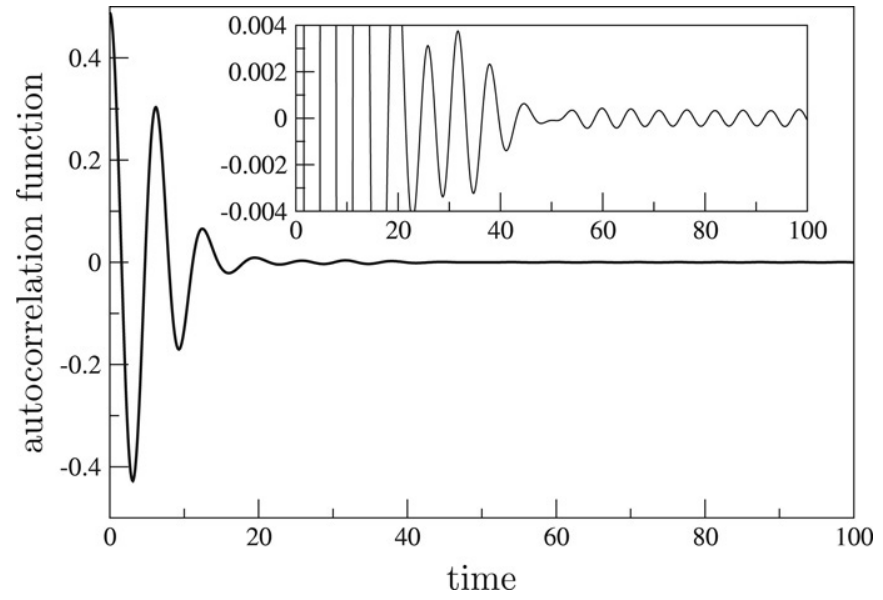

Fig. 15. Autocorrelation function of chaotic oscillations in the model B (the same parameters as in Fig. 11). The inset shows the correlation tail in an enlarged scale.

small contribution of this intrinsic amplitude regularity into the correlation function appears as a tail in Fig. 15.

\subsection{Model C: Arnold cat map in a perturbed heteroclinic cycle}

Let us start again with the system possessing the heteroclinic cycle (15) with $\omega_{1,2,3}=\omega_{0}$. This time we introduce additional coupling terms in the equations in such a way that each partial oscillator is driven by a product of variables of two other oscillators. We symmetrize the coupling terms to exclude the presence of the non-oscillating components, which could disturb the required phase transfer, and arrive at the following model C:

$\dot{x}_{1}=\omega_{0} y_{1}+\left(1-a_{1}-\frac{1}{2} a_{2}-2 a_{3}\right) x_{1}+\varepsilon\left(x_{2} y_{3}+x_{3} y_{2}\right)$,

$\dot{y}_{1}=-\omega_{0} x_{1}+\left(1-a_{1}-\frac{1}{2} a_{2}-2 a_{3}\right) y_{1}$,

$\dot{x}_{2}=\omega_{0} y_{2}+\left(1-a_{2}-\frac{1}{2} a_{3}-2 a_{1}\right) x_{2}+\varepsilon\left(x_{1} y_{3}+x_{3} y_{1}\right)$,

$\dot{y}_{2}=-\omega_{0} x_{2}+\left(1-a_{2}-\frac{1}{2} a_{3}-2 a_{1}\right) y_{2}$,

$\dot{x}_{3}=\omega_{0} y_{3}+\left(1-a_{3}-\frac{1}{2} a_{1}-2 a_{2}\right) x_{3}+\varepsilon\left(x_{2} y_{1}+x_{1} y_{2}\right)$,

$\dot{y}_{3}=-\omega_{0} x_{3}+\left(1-a_{3}-\frac{1}{2} a_{1}-2 a_{2}\right) y_{3}$,

where

$a_{1}=x_{1}^{2}+y_{1}^{2}, \quad a_{2}=x_{2}^{2}+y_{2}^{2}, \quad a_{3}=x_{3}^{2}+y_{3}^{2}$.

For this model we use set $\omega_{0}=1$ and $\varepsilon=0.03$. Again, trying 

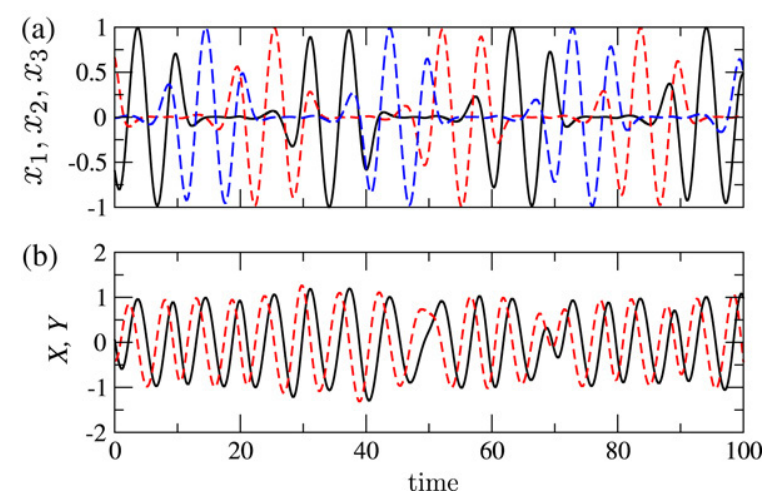

Fig. 16. (a): Time dependences for $x_{1}$ (black solid line), $x_{2}$ (red short dashed line), and $x_{3}$ (blue long dashed line), obtained from the computer solution of Eq. (19) for $\omega_{0}=1$ and $\varepsilon=0.03$. (b) Time dependencies of the symmetrized observables $X=x_{1}+x_{2}+x_{3}$ and $Y=y_{1}+y_{2}+y_{3}$. (For interpretation of the references to colour in this figure legend, the reader is referred to the web version of this article.)

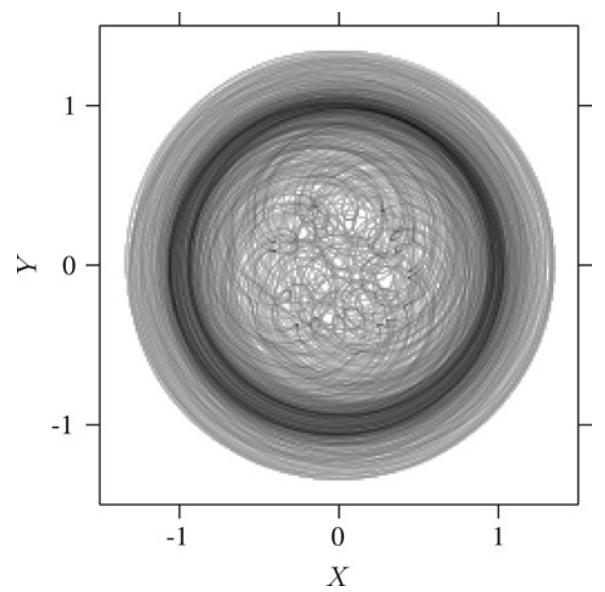

Fig. 17. Oscillations in system (19) for $\omega_{0}=1$ and $\varepsilon=0.03$ in a projection on the plane of the observables $(X, Y)$.

different initial conditions we always observed convergence of the orbits to one attractor.

Fig. 16 shows the evolution of the variables $x_{1}, x_{2}$, and $x_{3}$, as functions of time obtained from the computer solution of Eq. (19) after the decay of transients. In the course of time, three oscillators become excited in the order $1 \rightarrow 3 \rightarrow 2 \rightarrow$ $1 \rightarrow \cdots$. This whole process is not periodic. Symmetrized observables $X=x_{1}+x_{2}+x_{3}$ and $Y=y_{1}+y_{2}+y_{3}$ manifest oscillations with irregular phase and amplitude modulation; see the bottom panel in Fig. 16 and the phase portrait on the plane $(X, Y)$ in Fig. 17.

We can construct the Poincare map in the same way as described in the previous subsection, namely, in the sixdimensional phase space $\left\{x_{1}, y_{1}, x_{2}, y_{2}, x_{3}, y_{3}\right\}$ we define three surfaces determined by Eq. (18) and examine successive intersections of these surfaces by a trajectory. In this way we define a mapping $\mathbf{v}_{n+1}=\mathbf{T}\left(\mathbf{v}_{n}\right)$ : the pre-image is a fivedimensional vector $\mathbf{v}_{n}$ associated with a point on the surface $S_{j}=0$, and the image is a vector $\mathbf{v}_{n+1}$ on the next intersected surface $S_{k}=0$, which follows in accordance with the order
$S_{1} \rightarrow S_{3} \rightarrow S_{2} \rightarrow S_{1} \rightarrow \cdots$. Again, the spaces of images and pre-images may be identified due to a symmetry of the system.

Fig. 18 shows projections of the attractor in the Poincaré map onto three planes of the variables $\left(x_{j}, y_{j}\right)$ corresponding to three partial oscillators. Because of strong compression in the stable directions, the transversal structure of the attractor is not distinguishable in the panels (a) and (b), but may be seen in the panel (c).

For a sequence of successive intersections of the surfaces $S_{j}=0$ we define the phases of the oscillations as $\varphi_{n}=$ $\arg \left(x_{j}+\mathrm{i} y_{j}\right)$. Fig. 19 demonstrates that they obey the Fibonacci map (3) $\varphi_{n+1}=\varphi_{n}+\varphi_{n-1}+$ const with a good accuracy. As discussed in Section 2, the second iteration of the Fibonacci map yields the Arnold cat map. Fig. 20 illustrates the action of the map obtained from the numerical solution of Eq. (19) with a traditional picture of the cat face. It may be compared to Fig. 2 above.

The Lyapunov exponents for model (19) were calculated by the same algorithm as in the previous sections:

$$
\begin{aligned}
& \lambda_{1}=0.04786 \pm 0.00009, \\
& \lambda_{2}=-0.000001 \pm 0.00001, \\
& \lambda_{3}=-0.04840 \pm 0.00005, \\
& \lambda_{4}=-0.3184 \pm 0.0004, \\
& \lambda_{5}=-0.3673 \pm 0.0004, \\
& \lambda_{6}=-2.0000207 \pm 0.0000002 .
\end{aligned}
$$

There is one positive exponent, one zero, associated with a shift along the trajectory, and others negative.

Taking into account that the average time between the intersection of the surfaces $S_{j}=0$ at the given parameters is approximately $T_{\mathrm{av}}=9.943$, we can evaluate the Lyapunov exponents for the map $\mathbf{T}$ as $\Lambda=\lambda T$. The calculation yields $\rho_{1}=\exp \left[\Lambda_{1}\right] \approx 1.6094$ and $\rho_{3}=\exp \left[\Lambda_{3}\right] \approx 0.618$. The squared numbers $\rho_{1}^{2} \approx 2.59$ and $\rho_{3}^{2} \approx 0.382$ should be compared with the eigenvalues for the Arnold cat map (2) $(3+\sqrt{5}) / 2 \approx 2.618$ and $(3-\sqrt{5}) / 2 \approx 0.382$.

Note that for the Arnold map (2) a sum of the Lyapunov exponents vanishes. In our case, the attractor in the Poincaré map is concentrated close to a two-dimensional torus surface, the dynamics of which are governed approximately by the Arnold cat map. This assertion agrees with the fact that the sum of the largest two non-zero Lyapunov exponents is very small $\lambda_{1}+\lambda_{3} \approx 0.00054$. In fact, due to statistical errors it is hard to distinguish it from zero.

Therefore we conclude that the Lyapunov dimension of the attractor is nearly $D \approx 3$, and the dimension of the attractor in the Poincaré map is $d \approx 2$. This explains the visible absence of a fractal structure in Fig. 18(c)-(d).

Fig. 21 shows a plot of the autocorrelation function for the observable $X=x_{1}+x_{2}+x_{3}$ obtained in the computations. The correlations decay rather fast, without any visible tail.

\subsection{Model D: Hyperchaotic attractor in a perturbed hetero- clinic cycle}

Let us introduce a system with a hyperchaotic hyperbolic attractor. In contrast to two previous models, it will be non- 
(a)

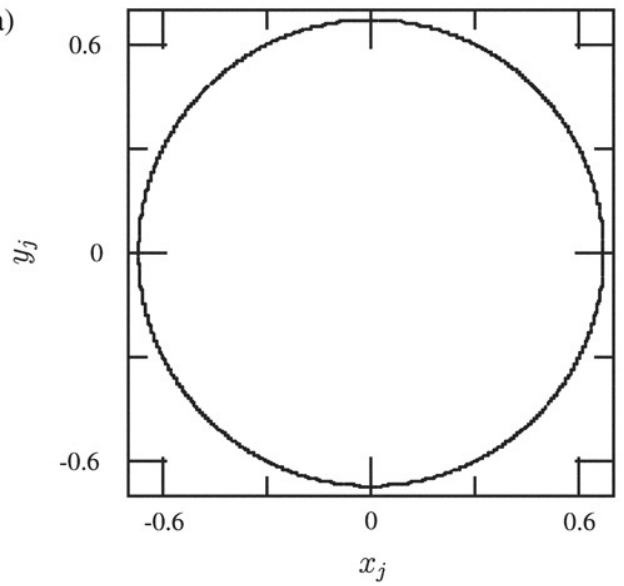

(c)

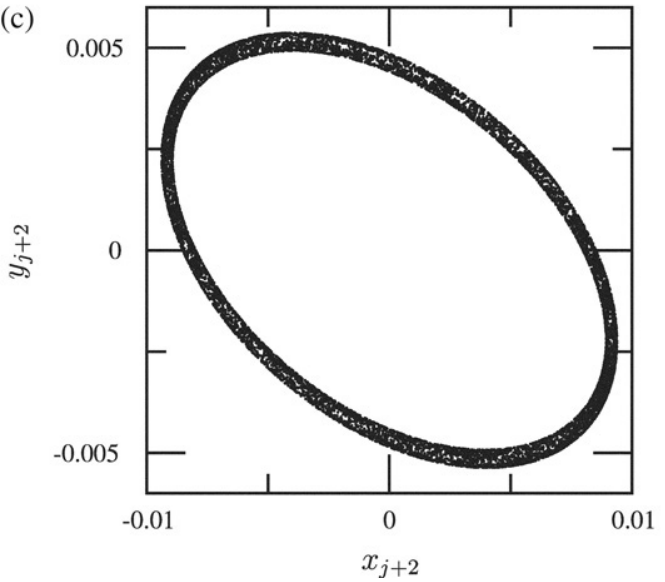

(b)

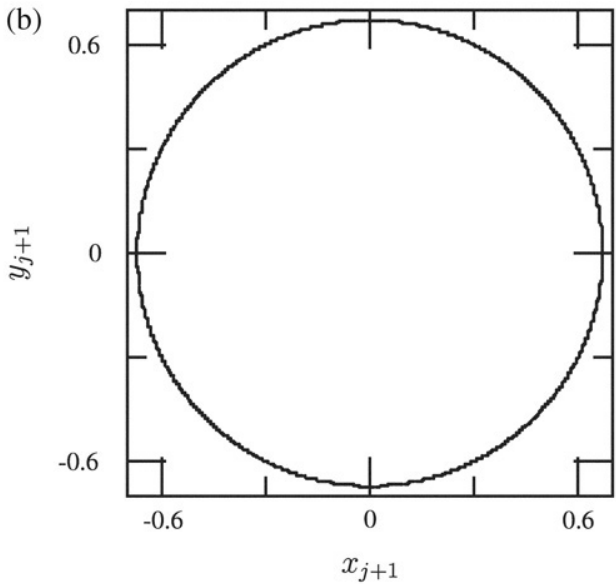

(d)

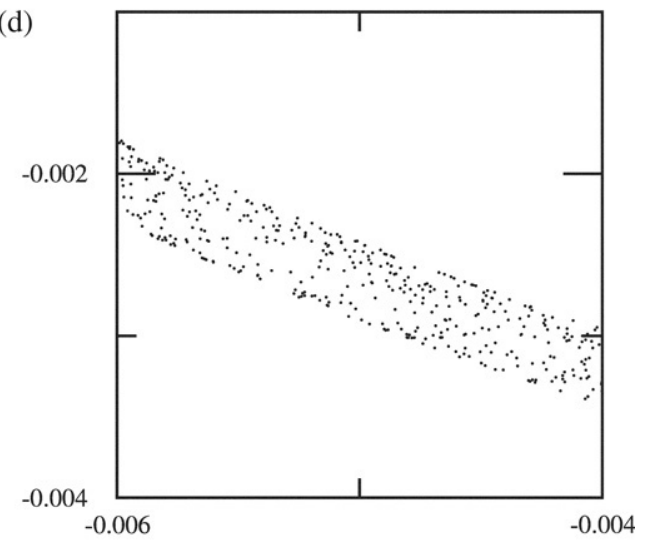

Fig. 18. Projections of the attractor for the Poincaré map of the model C (the same parameters as in Fig. 16) onto planes of the variables $\left(x_{j}, y_{j}\right)(\mathrm{a}),\left(x_{j+1}, y_{j+1}\right)$ (b), and $\left(x_{j+2}, y_{j+2}\right)$ (c) (notice that the indices should be taken modulo 3). Panel (d) shows an enlargement of a region of panel (c) to make the fine structure evident. Contrary to the case of model B above, the attractor looks not like a fractal.

symmetric. In the system with the heteroclinic cycle (15) we set $\omega_{1}=3 \omega_{0}, \omega_{2,3}=\omega_{0}$, and write down the following equations of the model D:

$\dot{x}_{1}=3 \omega_{0} y_{1}+\left(1-a_{1}-\frac{1}{2} a_{2}-2 a_{3}\right) x_{1}+\varepsilon_{1} x_{3}^{2} y_{3}$,

$\dot{y}_{1}=-3 \omega_{0} x_{1}+\left(1-a_{1}-\frac{1}{2} a_{2}-2 a_{3}\right) y_{1}$,

$\dot{x}_{2}=\omega_{0} y_{2}+\left(1-a_{2}-\frac{1}{2} a_{3}-2 a_{1}\right) x_{2}+\varepsilon_{2}\left(x_{1} y_{3}+x_{3} y_{1}\right)$,

$\dot{y}_{2}=-\omega_{0} x_{2}+\left(1-a_{2}-\frac{1}{2} a_{3}-2 a_{1}\right) y_{2}$,

$\dot{x}_{3}=\omega_{0} y_{3}+\left(1-a_{3}-\frac{1}{2} a_{1}-2 a_{2}\right) x_{3}+\varepsilon_{3} x_{2}$,

$\dot{y}_{3}=-\omega_{0} x_{3}+\left(1-a_{3}-\frac{1}{2} a_{1}-2 a_{2}\right) y_{3}$,

where

$a_{1}=x_{1}^{2}+y_{1}^{2}, \quad a_{2}=x_{2}^{2}+y_{2}^{2}, \quad a_{3}=x_{3}^{2}+y_{3}^{2}$,

and $\varepsilon_{1,2,3}$ are the coupling constants. Below we set $\omega_{0}=1$, $\varepsilon_{1}=\varepsilon_{3}=0.004, \varepsilon_{2}=0.1$. Starting with various initial conditions the system is observed to arrive always to one attractor.

As in the models $\mathrm{B}$ and $\mathrm{C}$, the partial oscillators become excited in the order $1 \rightarrow 3 \rightarrow 2 \rightarrow 1 \cdots$. At the moments in time where the conditions (18) are fulfilled, we may define the phases $\varphi_{j}$ of the rising oscillators as $\varphi_{j}=\arg \left(x_{j}+\mathrm{i} y_{j}\right)$. Let us examine how the transformations

$\varphi_{1} \rightarrow \varphi_{3} \rightarrow \varphi_{2} \rightarrow \bar{\varphi}_{1} \rightarrow \bar{\varphi}_{3} \rightarrow \bar{\varphi}_{2} \rightarrow \cdots$

look. Oscillator 1 is driven by the force proportional to $x_{3}^{2} y_{3}$. It contains the third harmonic, which is in resonance with the oscillator 1 and stimulates its excitation at the beginning of the epoch of activity. Hence, it adopts the phase

$\bar{\varphi}_{1}=3 \varphi_{3}+$ const.

Oscillator 3 in the course of the excitation is driven in resonance by the force proportional to $x_{2}$, so it adopts the phase from the oscillator 2:

$\bar{\varphi}_{3}=\varphi_{2}+$ const.

Finally, oscillator 2 is driven by the signal $\propto x_{1} y_{3}+x_{3} y_{1}$. At the beginning of the active epoch it is excited in a non-resonance manner (this is the reason why we assign a relatively large value 
(a)
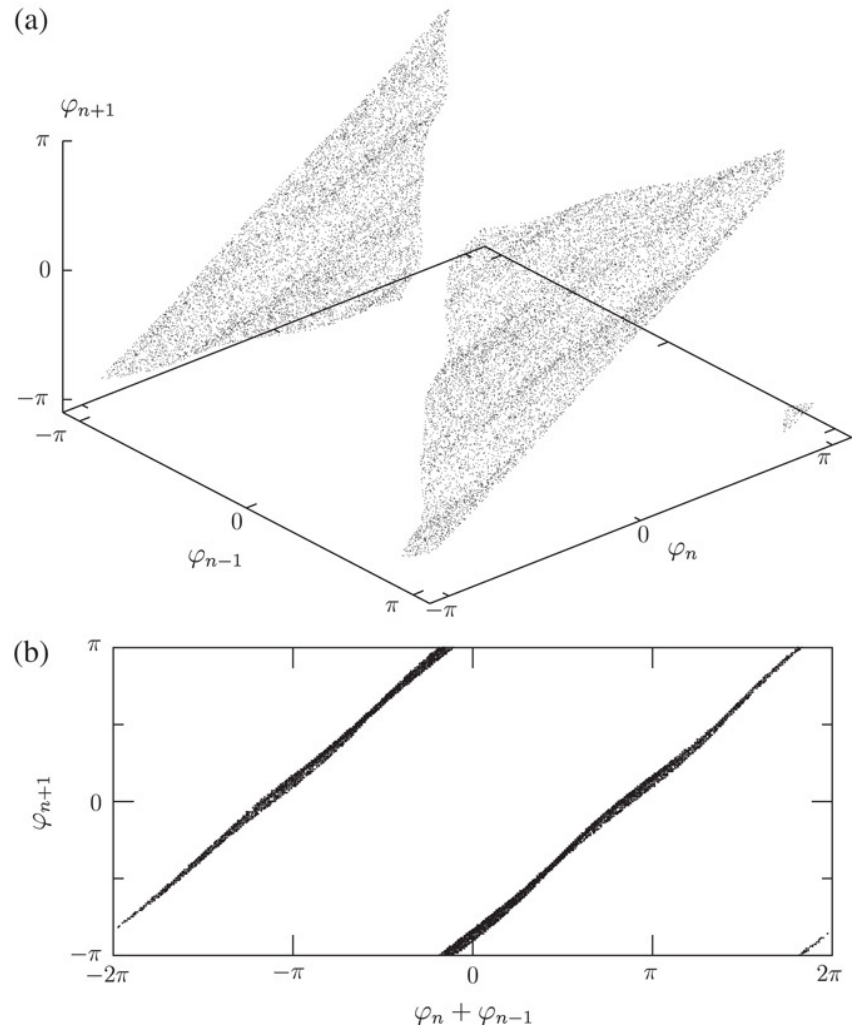

Fig. 19. (a) A three-dimensional representation of the dependence of $\varphi_{n+1}$ on $\varphi_{n}$ and $\varphi_{n-1}$ in model C with $\omega_{0}=1$ and $\varepsilon=0.03$. (b) Dependence of $\varphi_{n+1}$ on $\varphi_{n}+\varphi_{n-1}$.

for $\varepsilon_{2}$ ), and adopts the phase

$\bar{\varphi}_{2}=\varphi_{1}+\varphi_{3}+$ const.

Observe that these relations are equivalent to (5) up to a constant term. In Fig. 22 we demonstrate the empirical mappings obtained from the numerical simulation of the dynamics governed by Eq. (20).

The Lyapunov exponents for the model (20) evaluated from the computations are the following:

$\lambda_{1}=0.01878 \pm 0.00006$,

$\lambda_{2}=0.00621 \pm 0.00002$,

$\lambda_{3}=0.00000 \pm 0.000003$, $\lambda_{4}=-0.36803 \pm 0.0003$,

$\lambda_{5}=-0.43133 \pm 0.0003$,

$\lambda_{6}=-2.000026 \pm 0.000002$.

The presence of two positive Lyapunov exponents affirms the hyperchaotic nature of the dynamics. The third exponent is close to zero, linked with a perturbation associated with a shift along the orbit. Other exponents are negative that correspond to the approach of trajectories to the attractor.

Because all consecutive transformations $\varphi_{1} \rightarrow \varphi_{3} \rightarrow \varphi_{2} \rightarrow$ $\varphi_{1}$ are different, one should consider the overall transformation, e.g. with the surface of section $S_{1}$, as the Poincare map. The average time between successive cross-sections evaluated from the computations is $T_{\mathrm{av}} \approx 41.475=3 \cdot 13.825$. From nonzero exponents of the flow system, we estimate the Lyapunov exponents for the Poincaré map as $\Lambda=\lambda T_{\mathrm{av}}$. Observe that $\rho_{1}=\exp \left[T_{\mathrm{av}} \lambda_{1}\right] \approx 2.179$ and $\rho_{2}=\exp \left[T_{\mathrm{av}} \lambda_{2}\right] \approx 1.294$. In absolute values, these numbers are in good agreement with the eigenvalues of the hyperchaotic map (4); indeed, $(\sqrt{13}+1) / 2 \approx$ 2.303 and $(\sqrt{13}-1) / 2 \approx 1.303$. The Lyapunov dimension of the attractor estimated from the Kaplan-Yorke formula is 3.068.

We illustrate the time evolution of the oscillators in Fig. 23, and the phase portrait in the symmetrized observables $X=$ $x_{1}+x_{2}+x_{3}$ and $Y=y_{1}+y_{2}+y_{3}$ is depicted in Fig. 24. The autocorrelation function of the observable $X=x_{1}+x_{2}+x_{3}$ is shown in Fig. 25. All these diagrams demonstrate the good chaotic properties of the system (20).

\section{Conclusion}

The problem of constructing simple flow systems with hyperbolic chaotic attractors appears to be of general interest both from the fundamental and practical points of view. Such examples would be important illustrations for the welldeveloped mathematical theory of the hyperbolic systems.

In this paper, we present an approach based on the use of coupled self-sustained oscillators with alternating excitation. An additional coupling allows us to transfer the phases from one partner to another, and simultaneously to transform them according to a desired chaotic map on a circle or on a torus. This idea of the phase manipulation for constructing the hyperbolic
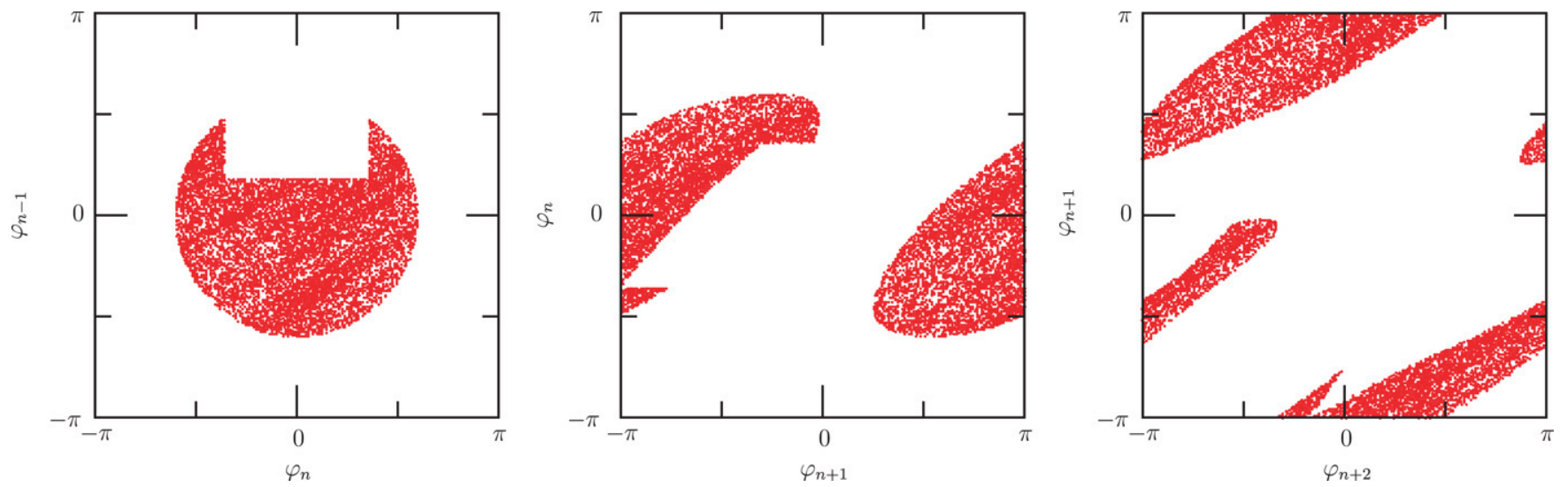

Fig. 20. Diagram for the phases of successively exciting partial oscillators in the model $\mathrm{C}$ with $\omega_{0}=1$ and $\varepsilon=0.03$, with the use of a picture of the cat face. 
chaotic dynamics is adopted from earlier works [8,9]. Here we present for the first time examples of autonomous systems with apparently hyperbolic attractors of different types. One of the constructed models delivers an example of a system of minimal possible dimension possessing the attractor of Smale-Williams type. Methodologically, a new element in comparison with Refs. [8,9] is to employ a non-resonance transfer of the excitation between the oscillators.

In our analysis of the suggested models we start with a qualitative consideration, and subsequently perform a careful computer numerical study. An important component in each case is a visual inspection of diagrams illustrating the phase transfer, to be sure that it belongs to the desirable class, like the expanding circle map or the hyperbolic toral maps. Numerical studies include the calculation of the spectrum of the Lyapunov exponents, positive exponents are then compared to those in the underlying chaotic maps.

From our experience of the performed numerical studies, we can say that the chaotic attractors observed in our models surely look structurally stable being insensitive to concrete selection of the parameters. For example, in the model A the coupling parameter $\varepsilon$ may be varied in an interval at least from 0.0004 to 0.3 , and it does not change neither nature of the attractor nor the approximate value of the largest Lyapunov exponent for the Poincaré map. The same is true in respect to the variation of other parameters, at least in an interval about, say, $\pm 10 \%$ of the presented value. In particular, the frequency detuning may be introduced without any destructive consequences. The particular form of combinations of variables in the coupling terms is relevant, in agreement with the argumentation developed in Section 3. It is, however, of no significance, whether the coupling is introduced in the first, or the second, or in both equations for a partial oscillator. It seems that in the parameter range under consideration the attractor is unique, as far as we can judge from the numerical studies. Analogous observations hold also for the models B, C, and D.

An important issue we do not address in this paper relates to possible bifurcation scenarios of the onset of hyperbolic strange attractors, e.g. when applied to our models A, B, C, D. Mathematical studies of which we are aware seem insufficient in this respect.

Firstly, recall the so-called "Ruelle-Takens scenario" widely discussed in the 1970s-80s. In fact, it does not clarify concrete relevant bifurcation routs, although a possibility was proven of appearance of hyperbolic strange attractors from perturbations of quasiperiodic motions on tori of dimension of 3 and more [3].

A consideration of codimension one bifurcations generating the hyperbolic attractors by Shilnikov and Turaev [6] (cf. [15]) perhaps deserves attention in the context of our research. It is remarkable that they incorporate three types of hyperbolic attractor similar to those discussed in our paper. Nevertheless, these studies scarcely help in an understanding of mechanisms of birth of the hyperbolic attractors in our models. Indeed, the blue-sky-catastrophe phenomena, including an unbounded growth of the characteristic timescale at the threshold of the disappearance of the attractors, are not observed in our models,

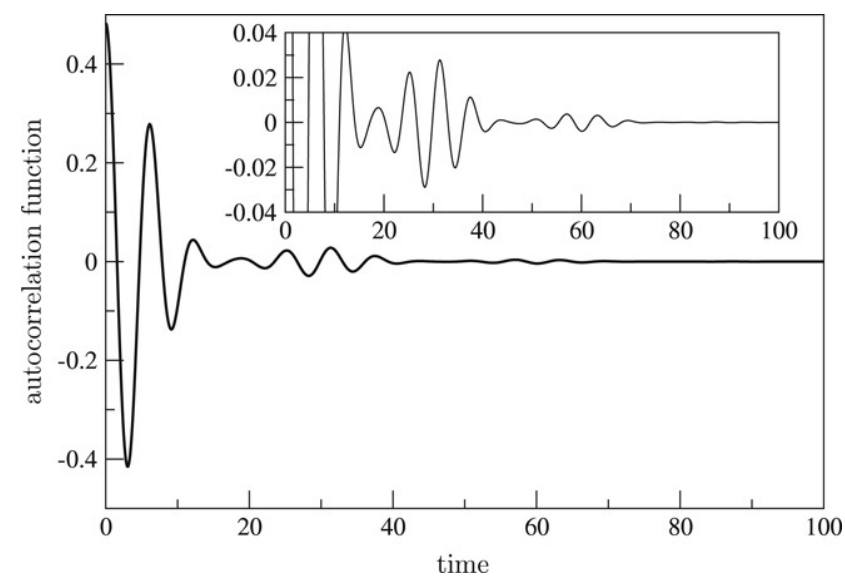

Fig. 21. Autocorrelation function of chaotic oscillations in the model $\mathrm{C}$ with $\omega_{0}=1$ and $\varepsilon=0.03$. The inset shows the correlation tail in an enlarged scale.

at least without some essential modification of them. Moreover, in the Shilnikov and Turaev construction, the appearance of the relevant topological property on the map for the angle coordinate during the "flight-time" dynamics between two cross-sections in the phase space (see, e.g., Fig. 15 in the paper [6]) is simply pre-assumed, leaving unanswered the question of the mechanisms responsible for this property. In fact, these mechanisms remain outside the problematics associated with the saddle-node-like phenomena referred to as the blue-sky catastrophes. Conversely, these mechanisms are for us the focus of attention.

Our computations show that the hyperbolic strange attractors do exist in some ranges of parameter, but certainly cease to exist (at least in the old form) when the variations of parameters become sufficiently large. Usually in such cases, we observe complex objects that we still cannot classify and characterize. We leave this task for future research.

As is observed in the calculations, the correlation functions for our models in some cases manifest long-range tails of a relatively small magnitude. We explain this by the presence of an almost regular alternating evolution of the amplitudes of coupled oscillators, while strong chaotization reveals itself in the dynamics of the phases. In general, correlations in continuous-time systems with hyperbolic attractors must not demonstrate a perfect decay, and what we observe are examples of such behavior. A detailed study of these correlation tails will be published elsewhere.

Of course, a more rigorous mathematical treatment of the suggested models is desirable. One possibility is to apply a technique of computer verification of conditions of hyperbolicity in terms of inclusions for expanding and contracting cones in the tangent spaces at different points of the attractor or in an absorbing domain containing the attractor (In Ref. [16] this approach was applied to a non-autonomous system possessing a hyperbolic attractor.). Preliminary calculations, performed by I. R. Sataev (private communication) confirm the hyperbolicity conditions for the attractor in our model $\mathrm{A}$ at some particular parameter values. It seems much more difficult to carry out analogous computations for models B, C, D because of higher phase-space dimensions. 

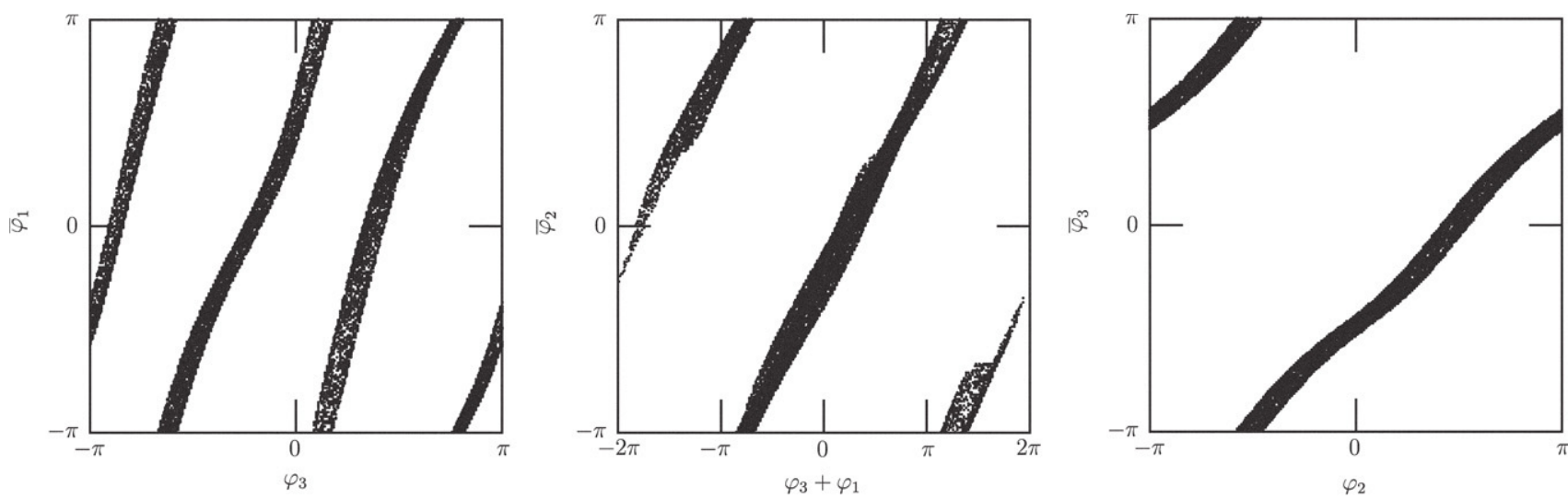

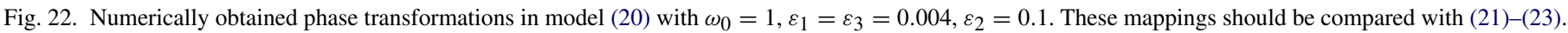
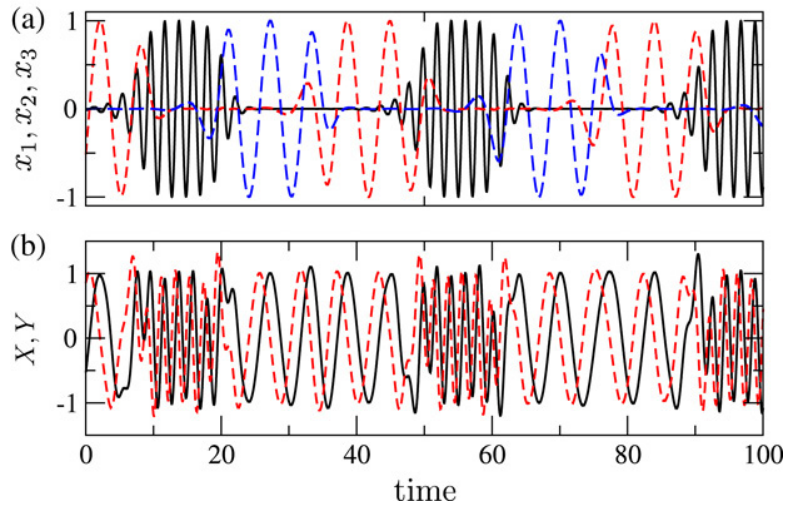

Fig. 23. (a): Time dependences for $x_{1}$ (black solid line), $x_{2}$ (red short dashed line), and $x_{3}$ (blue long dashed line), obtained from the computer solution of Eq. (20) with $\omega_{0}=1, \varepsilon_{1}=\varepsilon_{3}=0.004, \varepsilon_{2}=0.1$. Notice that the first oscillator has a frequency three times greater than the other two. (b) Time dependencies of the symmetrized observables $X=x_{1}+x_{2}+x_{3}$ and $Y=y_{1}+y_{2}+y_{3}$. (For interpretation of the references to colour in this figure legend, the reader is referred to the web version of this article.)

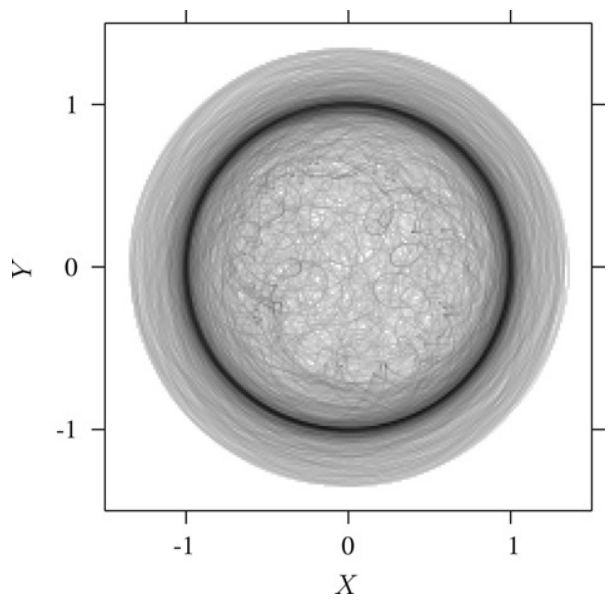

Fig. 24. Oscillations in system (20) with $\omega_{0}=1, \varepsilon_{1}=\varepsilon_{3}=0.004, \varepsilon_{2}=0.1$ in a projection on the plane of observables $(X, Y)$.

Generalization of the heteroclinic cycle construction for systems of more than three oscillators may give rise to more far-reaching possibilities for the phase manipulations; that could result in constructing hyperbolic chaotic attractors with

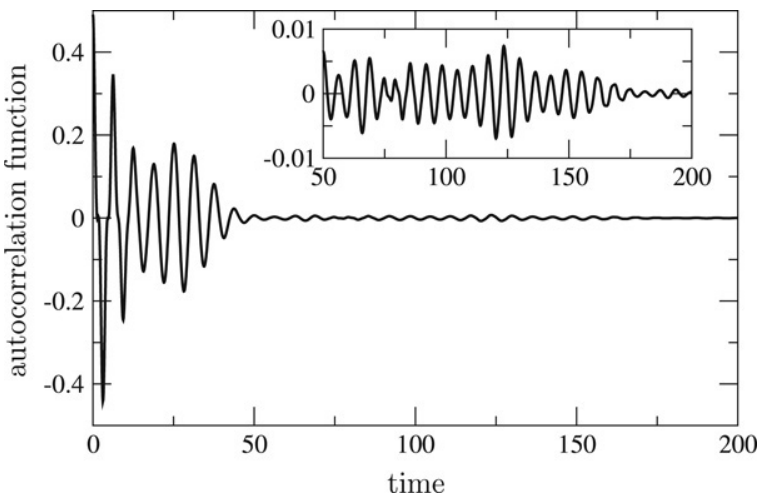

Fig. 25. Autocorrelation function of chaotic oscillations in the model D with the same parameter values as in Figs. 22-24. The inset shows the correlation tail in an enlarged scale.

stronger chaotic properties (larger number of positive Lyapunov exponents, large values of them, fast decaying correlations, broad frequency spectra).

We expect that the examples of hyperbolic attractors we have introduced, or similar models, may be implemented as real devices, e.g. in electronics, mechanics, optics. Contrary to many other systems with chaotic behavior, the hyperbolic strange attractors are structurally stable (robust). Such systems may be useful for applications, e.g. as noise generators, or as transmitters in chaos-based communications.

\section{Acknowledgments}

We thank V.N. Belykh and I.R. Sataev for their illuminating discussions. The work was supported by grants of DFG and RFBR (No 04-02-04011 and 06-02-16619).

\section{References}

[1] A. Katok, B. Hasselblatt, Introduction to the Modern Theory of Dynamical Systems, Cambridge University Press, 1995.

[2] R. Plykin, Sources and sinks for A-diffeomorphismes of surfaces, Math. USSR, Sb. 23 (1974) 233-253.

[3] S. Newhouse, D. Ruelle, F. Takens, Occurrence of strange axiom A attractors near quasi periodic flows on $T^{m}, m \geq 3$, Comm. Math. Phys. 64 (1978) 35-40.

[4] T. Hunt, Low dimensional dynamics: Bifurcations of Cantori and realisations of uniform hyperbolicity. Ph.D. Thesis, University of Cambridge, 2000. 
[5] V.N. Belykh, I.V. Belykh, E. Mosekilde, Hyperbolic Plykin attractor can exist in neuron models, Int. J. Bifurc. Chaos 15 (2005) 3567-3578.

[6] L.P. Shilnikov, D.V. Turaev, Simple bifurcations leading to hyperbolic attractors, Comput. Math. Appl. 34 (2-4) (1997) 173-193.

[7] L.P. Shilnikov, Mathematical problems of nonlinear dynamics: A tutorial, Int. J. Bifurc. Chaos 7 (1997) 1353-2001.

[8] S.P. Kuznetsov, Example of a physical system with a hyperbolic attractor of the Smale-Williams type, Phys. Rev. Lett. 95 (2005) 144101.

[9] S.P. Kuznetsov, E.P. Seleznev, A strange attractor of the Smale-Williams type in the chaotic dynamics of a physical system, J. Exp. Theor. Phys. 102 (2) (2006) 355-364.

[10] O.B. Isaeva, A.Yu. Jalnine, S.P. Kuznetsov, Arnold's cat map dynamics in a system of coupled non-autonomous van der Pol oscillators, Phys. Rev. E 74 (2006) 046207.

[11] A.Yu. Jalnine, S.P. Kuznetsov, On a possibility of realization of a strange nonchaotic attractor of Hunt and Ott in a physical system, Tech. Phys. 52 (2007) 401-408.

[12] P. Gaspard, Maps. Encyclopedia of Nonlinear Science, Routledge, New York, 548-553, 2005.

[13] J. Guckenheimer, P. Holmes, Structurally stable heteroclinic cycles, Math. Proc. Cambridge Philos. Soc. 103 (1988) 189-192.

[14] E. Ott, Chaos in Dynamical Systems, Cambridge Univ. Press, Cambridge, 1992.

[15] D. Pazó, M.A. Mathías, Direct transition to high-dimensional chaos through a global bifurcation, Europhys. Lett. 72 (2) (2005) 176-182.

[16] S.P. Kuznetsov, I.R. Sataev, Hyperbolic attractor in a system of coupled non-autonomous van der Pol oscillators: Numerical test for expanding and contracting cones, Phys. Lett. A 365 (2007) 97-104. 